\title{
PPARG in osteocytes is essential for sclerostin expression, bone mass, marrow adiposity and TZD-induced bone loss
}

Sudipta Baroi ${ }^{1,3}$, Piotr J. Czernik ${ }^{2}$, Amit Chougule ${ }^{1,3}$, Patrick R. Griffin ${ }^{4}$, Beata Lecka-Czernik ${ }^{1,2,3}$

${ }^{1}$ Department of Orthopaedic Surgery, ${ }^{2}$ Department of Physiology and Pharmacology, ${ }^{3}$ Center for Diabetes and Endocrine Research, University of Toledo, College of Medicine and Life Sciences, Toledo, OH. ${ }^{4}$ The Scripps Research Institute, Jupiter, FL.

Running title: Osteocytic PPARG regulates sclerostin and BMAT

Address to correspondence:

Beata Lecka-Czernik, PhD, Department of Orthopaedic Surgery, Mail Stop 1008, College of Medicine and Life Sciences, University of Toledo, Toledo, Ohio 43614

Phone: 419-383-4140

Beata.Leckaczernik@utoledo.edu

Key words: PPARG, SOST/sclerostin, PPRE, bone mass, marrow adipocytes 


\begin{abstract}
PPARG role in regulation of osteocyte function is largely unknown. We report that PPARG is essential for sclerostin production, a recently approved target to treat osteoporosis. There is an excellent correlation in osteocytes between Sost/sclerostin and PPARG at the transcript and protein levels, and increased bone mass in mice with osteocyte-specific deletion of PPARG $\left(\gamma \mathrm{OT}^{\mathrm{KO}}\right)$ correlated with increased WNT signaling and bone forming activity of endosteal osteoblasts and decreased marrow fat. The $8 \mathrm{~kb}$ sequence upstream of Sost gene transcription start site possesses multiple PPARG binding elements (PPREs) with at least two of them binding PPARG with dynamics reflecting its activation and the levels of Sost transcript and sclerostin protein expression. Older $\gamma \mathrm{OT}^{\mathrm{KO}}$ female mice are largely protected from TZD-induced bone loss providing proof of concept that PPARG in osteocytes can be pharmacologically targeted. Our study opens the possibility to consider repurposing PPARG as a target for treatment of osteoporosis.
\end{abstract}




\section{Introduction}

Growing number of compelling evidences point to the mechanistic and functional connection between energy metabolism and bone mass (1-3). Peroxisome proliferator-activated receptor gamma (PPARG) exemplifies this connection. PPARG is a transcription factor that regulates network of genes associated with glucose and lipid metabolism, insulin signaling, adipocyte differentiation, and inflammation. PPARG has been implicated in the pathology of numerous diseases like diabetes, obesity, cancer, and atherosclerosis (4-6). In bone, PPARG controls osteoblasts and adipocytes differentiation from bone marrow stroma cells (BMSC) $(7,8)$, and osteoclasts differentiation from cells of hematopoietic lineage, monocytes (9). Increased activity of PPARG in response to pharmacologic treatment with TZDs, PPARG full agonists and insulin sensitizers, results in bone loss associated with accumulation of fat in bone marrow, and up to two-fold increase in fracture rate in older women with diabetes $(3,10,11)$. Activated PPARG increases osteoclast resorptive activities, and is skewing BMSC lineage allocation toward adipocytes and away from osteoblasts, which at least in part explains PPARG negative effects on bone. Our previous studies have indicated that there is an in vivo relationship between PPARG activity and Sost expression in osteocytes, and most importantly that the bone anabolic effect of a new class of insulin sensitizers, which act as PPARG inverse agonists and block S112 dephosphorylation, is associated with decreased Sost expression (12). These findings prompted us to explore the link between PPARG activity and sclerostin production with systematic and mechanistic approach.

Produced in osteocytes, the most abundant cells in bone, sclerostin protein regulates bone formation and resorption via inhibition of WNT signaling and production of RANKL (13). The newly approved anti-osteoporotic therapy with romosozumab consists of a monoclonal antibody 
targeting sclerostin. Romosozumab is highly efficacious with respect to the anabolic effect on bone and fracture prevention in postmenopausal women; however, there are safety concerns caused by the growing number of reported serious cardiovascular (CV) effects among the users of this drug, especially among individuals with high risk for CV events (14-18). Although the exact etiology of romosozumab-triggered CV effects is not determined, the off-target effects of the drug may include sclerostin produced in vascular endothelium and heart valves (19), and animal studies suggest that sclerostin produced by endothelium have a protective effect against vascular calcification (20). The growing list of potential, although not yet clinically recognized off-target effects, includes also joint diseases. It has been shown that sclerostin improves posttraumatic osteoarthritis (21) and that sclerostin produced by synovial fibroblasts have an inhibitory effect on progression of the TNF $\alpha$-dependent rheumatoid arthritis, which is exacerbated with anti-sclerostin antibody therapy (22). Based on the above concerns and the superior anti-osteoporotic effects of anti-sclerostin therapy, the transcriptional control of sclerostin production in osteocytes can be a great alternative approach to the antibody therapy, especially when the transcription factor has been already identified as a therapeutic target, which is a case for PPARG.

Thus, with our long-term objective to demonstrate the feasibility of therapeutic targeting of PPARG to treat simultaneously diabetes, diabetic bone disease, and osteoporosis we designed current study to understand the role of PPARG in regulation of osteocyte function. To achieve this goal, we have developed mouse model of osteocyte-specific deletion of PPARG using DmplCre driver and Ppary loxP system. Here, we report that PPARG in osteocytes is a viable target to regulate sclerostin levels specifically in bone, and as a consequence bone mass and marrow adiposity, and skeletal response to pharmacologic activators of PPARG. Sclerostin 
is transcriptionally regulated by PPARG and PPARG is essential for sclerostin expression. These findings support consideration of selective pharmacological targeting of PPARG in osteocytes to control sclerostin production.

\section{Results}

\section{Ppary is highly expressed in osteocytes, and its expression increases with aging and with rosiglitazone treatment.}

Ppary expression was analyzed in femora cortical bone fractions highly enriched in either osteocytes or endosteal osteoblasts, as validated at the level of cell type specific gene markers expression (Suppl. Fig.S1). As shown in Fig. 1A, an expression of Ppary in osteocytes isolated from 6 mo old C57BL/6 males was 8-fold higher than the expression in endosteal osteoblasts isolated from the same femora cortical bone, whereas in 6 mo old females osteocyte expression of Ppary was 40-fold higher than in osteoblasts. Interestingly, Ppary mRNA expression increases with aging, as osteocytes derived from 10 mo old females have 3-fold higher Ppary levels than osteocytes derived from 6 mo old females (Fig. 1B). Finally, Ppary mRNA expression increases in osteocytes of mice treated with TZD rosiglitazone (Fig. 1C) indicating that PPARG protein in osteocytes is functional, because it responds to activation with the high affinity ligand by autoregulating its own expression, a phenomenon well documented in cells of adipocytic lineage (4).

These findings gave us strong bases for seeking the role of PPARG nuclear receptor in osteocytes. Mice with PPARG deficiency primarily in osteocytes, the $\gamma \mathrm{OT}^{\mathrm{KO}}$ mice, were constructed by crossing Ppary ${ }^{\text {flfl }}$ with $10 \mathrm{~kb} \mathrm{Dmpl}^{\mathrm{Cre}}$ strains to remove exon 1 and exon 2 from the Ppary gene, as shown in Fig. 1D. Offspring with a desirable $\gamma \mathrm{OT}^{\mathrm{KO}}$ genotype are viable and 
born with predicted Mendelian distribution with respect to sex and genotype. In all experiments, control mice $(\mathrm{Ctrl})$ consist of littermates with either $D m p^{\mathrm{Cre}} P p a r \gamma^{\mathrm{WT}}$ or Dmp ${ }^{\mathrm{WT}} P_{p a r \gamma} \gamma^{\mathrm{fl} / \mathrm{fl}}$

genotypes to assure for a random distribution of parental genetic background.

A

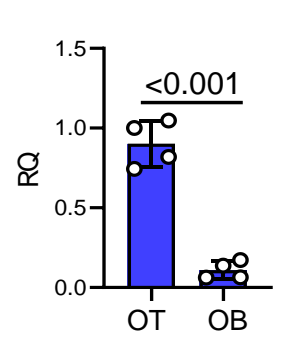

B

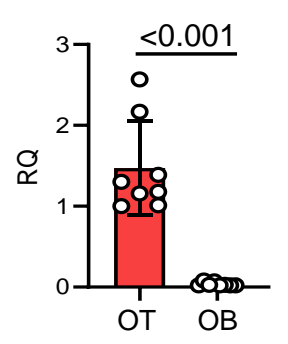

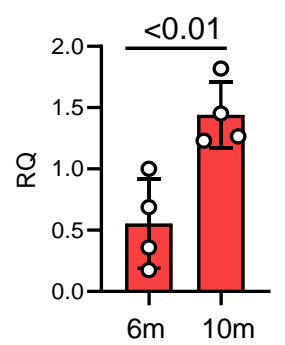

C

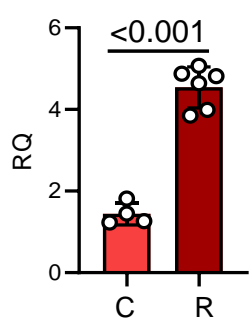

D

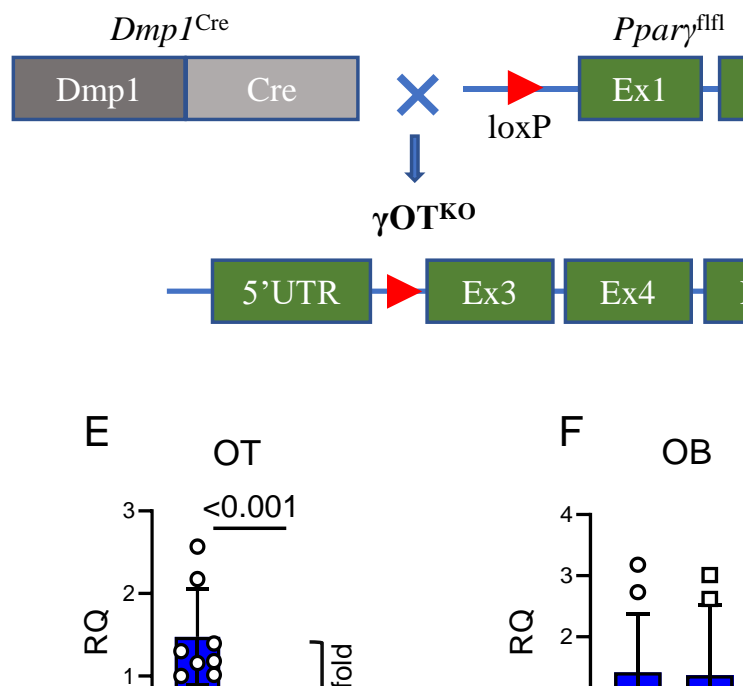

Figure 1. Development of $\gamma \mathrm{OT}^{\mathrm{KO}}$ mice. A. Ppary is highly expressed osteocytes (OT) as compared to osteoblasts (OB). OT and OB were isolated from femora cortical bone of $6.5-7$ mo old C57BL/6 males (blue) $\left(\gamma \mathrm{OT}^{\mathrm{KO}} \mathrm{n}=4\right.$ and $\left.\mathrm{Ctrl} \mathrm{n}=4\right)$ and females (red) $\left(\gamma \mathrm{OT}{ }^{\mathrm{KO}} \mathrm{n}=8\right.$ and Ctrl $\left.\mathrm{n}=8\right)$ using a method of differential collagen digestion and immediately processed for RNA isolation. B. Ppary expression in OT increases with aging. OT were isolated as above from femora of 6 mo and 10 mo old C57BL/6 female mice. C. Rosiglitazone treatment increases expression of osteocytic Ppary. OT were isolated from 10 mo old C57BL/6 female mice treated with $25 \mathrm{mg} / \mathrm{kg} / \mathrm{d}$ rosiglitazone $(\mathrm{R})$ for 6 weeks $\left(\gamma \mathrm{OT}{ }_{\mathrm{n}=6}^{\mathrm{KO}}\right.$ and $\mathrm{Ctrl}$ $\mathrm{n}=4)$. D. Schematic of $\gamma \mathrm{OT}{ }^{\mathrm{KO}}$ mice development. $\gamma \mathrm{OT}^{\mathrm{KO}}$ mice have deleted exon 1 and 2 from Ppar $\gamma$ gene, as a result of crossing $D m p l^{\text {Cre }}$ and $P p a r \gamma^{\text {flfl }}$ mice. E. Ppary expression in OT isolated from 6 mo old $\gamma \mathrm{OT}^{\mathrm{KO}}(\mathrm{n}=6)$ and $\mathrm{Ctrl}(\mathrm{n}=8)$ male mice. F. Ppary expression in OB isolated from the same mice as in E. Numbers above the horizontal bars indicate $\mathrm{p}$ values calculated with parametric unpaired Student $\mathrm{t}$ test. RQ - relative quantity. 
The $\gamma \mathrm{OT}^{\mathrm{KO}}$ model was validated on several levels. The expression of Ppary in osteocytes isolated from femora of $\gamma \mathrm{OT}^{\mathrm{KO}}$ mice was 100 -fold lower than the expression in osteocytes isolated from Ctrl mice (Fig. 1E), while Ppary expression in osteoblasts isolated from $\gamma \mathrm{OT}^{\mathrm{KO}}$ mice was not affected, as compared to Ctrl mice (Fig. 1F). Since there is a concern that Cre recombinase under control of $10 \mathrm{~kb} \mathrm{Dmpl}$ promoter can be activated in other than osteocyte cell types, we analyzed the level of Ppary expression in different tissues of adult $\gamma \mathrm{OT}^{\mathrm{KO}}$ and $\mathrm{Ctrl}$ mice. As shown in the Suppl. Table S1, we did not detect significant differences in Ppary expression in muscle, bone marrow, liver, WAT, BAT, kidney, cerebellum, and small intestine of Ctrl and $\gamma \mathrm{OT}^{\mathrm{KO}}$ suggesting that even if $10 \mathrm{~kb} \mathrm{Dmpl}{ }^{\mathrm{Cre}}$ construct is "leaky", the expression of Ppary in other tissues of adult $\mathrm{\gamma OT}^{\mathrm{KO}}$ mice is not obviously affected, which validates our study on the role of PPARG in osteocytes of mature bone.

\section{PPARG transcription factor is essential for sclerostin production}

The level of PPARG protein deletion in osteocytes is paralleled by the level of sclerostin protein produced in these cells (Fig. $2 \mathrm{~A}$ and $2 \mathrm{~B}$ ). There is an excellent correlation $\left(\mathrm{R}^{2}=0.98\right)$ between PPARG and sclerostin levels, with both proteins being significantly decreased in osteocytes isolated from bone of $\gamma \mathrm{OT}^{\mathrm{KO}}$ mice (Fig. 2C). An analysis of sera of the same $\gamma \mathrm{OT}{ }^{\mathrm{KO}}$ mice, show tendency to decrease in sclerostin levels but did not parallel a dramatic decrease in sclerostin protein in bone (Fig. 2D). These suggest that sclerostin levels in circulation are not in direct correlation with its production in bone and point to other sources for sclerostin production. Indeed, a number of studies indicates other organs such kidney, aorta and testes as producing sclerostin (23). 
A

$\begin{array}{lll}\text { үOTKO } & & \text { Ctrl } \\ 12 & 2\end{array}$

PPARG

$\beta$-actin

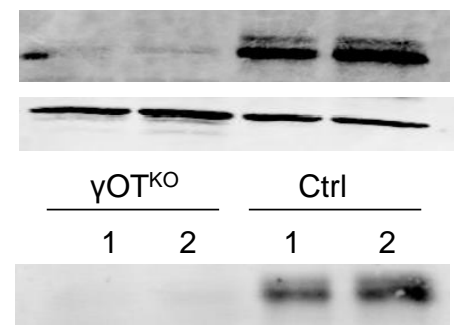

Sclerostin

$\beta$-actin

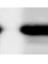

C

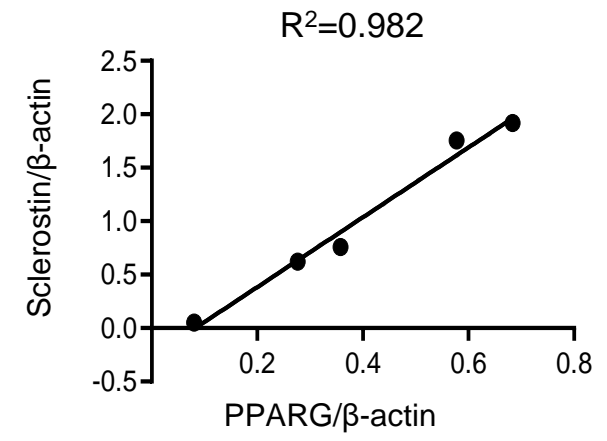

B

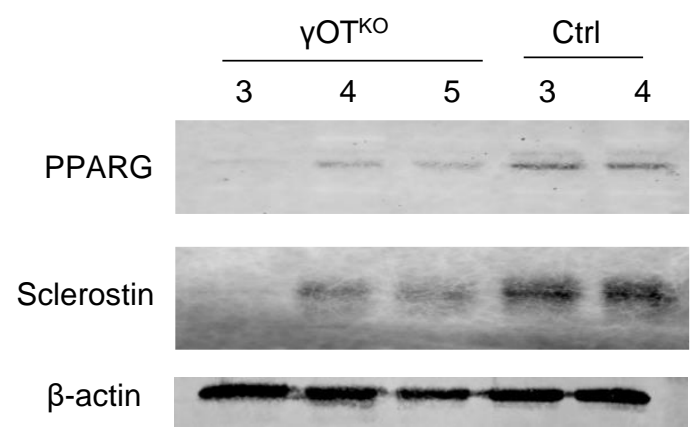

$\mathrm{D}$

Figure 2. Correlation between PPARG and sclerostin levels in osteocytes. A. and B. Levels of PPARG and sclerostin proteins in OT isolated from 6.5 mo old male $\gamma \mathrm{OT}{ }^{\mathrm{KO}}$ and $\mathrm{Ctrl}$ mice and either analyzed on separate (A) or the same (B) Western blot membrane. $\left(\gamma \mathrm{OT}{ }_{n=5 ;}^{\mathrm{KO}} \mathrm{Ctrl} n=4\right)$. C) Pearson correlation analysis of PPARG and sclerostin protein levels shown in panel B. Coefficient of determination $\left(\mathrm{R}^{2}=0.9821\right)$ was calculated based on bands density assessed by Image $\mathrm{J}$ and normalized to b-actin. $\mathrm{D}$. Sclerostin levels measured by ELISA in sera of $\gamma_{\mathrm{OT}}{ }^{\mathrm{KO}}(\mathrm{n}=4)$ and $\mathrm{Ctrl}(\mathrm{n}=7) 6$ mo old male mice. Statistical significance was calculated using parametric unpaired Student's t test.

\section{Identification of PPREs located upstream of Sost transcription start site}

The $8 \mathrm{~kb}$ DNA sequence upstream of transcription start site (TSS) of Sost gene was analyzed using a position weighted matrix (PWM) for prediction of binding sites for mouse PPARG::RXRA complex. The analysis was performed with assistance of the JASPAR database for curated, non-redundant set of transcription factor binding sites profiles. The position matrix 
algorithm is based on over 850 murine DNA sequences that were identified to bind the PPARG::RXRA heterodimer (Suppl. Fig. S2). Based on PWM, the JASPAR projected 15 PPARG response elements (PPRE) in the Sost gene promoter/enhancer region (Table 1 and Fig. 3A). The PPREs are located on both DNA strands. In the proximal $2 \mathrm{~kb}$ region, which represents Sost promoter, there are 4 PPREs, two of them with relatively low scores for PPARG binding consensus sequence are located within $1 \mathrm{~kb}$ from TSS (PPRE1 and PPRE2) and the other two (PPRE3 and PPRE4) with higher scores at 1.8 - 1.9 kb distance from TSS. A cluster of 7 PPREs (PPRE5 to PPRE12) has been detected within $1 \mathrm{~kb}$ fragment at the $3.5-4.5 \mathrm{~kb}$ distance from TSS. Binding scores for PPREs in this cluster are high and varied from 8.1 to 12.5 . The last cluster, with relatively high scores, consists of 3 PPREs (PPRE13 to PPRE15) and is located at the 7 - $8 \mathrm{~kb}$ upstream from TSS (Fig. 3A and Table 1). For further analysis, we selected two PPRE-containing regions based on their location and the highest scores for the consensus of PPARG binding sequence. The proximal PPRE3 located in the promoter region and two distal PPRE14 an PPRE15 located in the potential enhancer region. The consensus score for PPARG binding is 9.2 for PPRE3, and 15.3 and 9.6 for PPRE14 and PPRE15, respectively (Table 1).

Using the chromatin immunoprecipitation assays (ChIP), we tested selected PPREs for their binding PPARG in basal conditions and upon activation with rosiglitazone. The assay was performed on osteocyte-like MLO-Y4 cells and showed that PPARG binds to both locations of the PPRE motifs (Fig. 3B). In basal conditions, PPARG protein occupies proximal PPRE3 and its binding to the PPRE14/15 location is rather weak. Activation with rosiglitazone slightly increases binding to PPRE3, while treatment with the covalent antagonist GW9662 significantly decreases PPARG binding to this site (Fig. 3B and Suppl. Fig. S3). In contrast, treatment with rosiglitazone induces robust recruitment of PPARG to PPRE14/15 binding sites, which is 
completely abrogated in the presence of GW9662 antagonist (Fig. 3B).

A

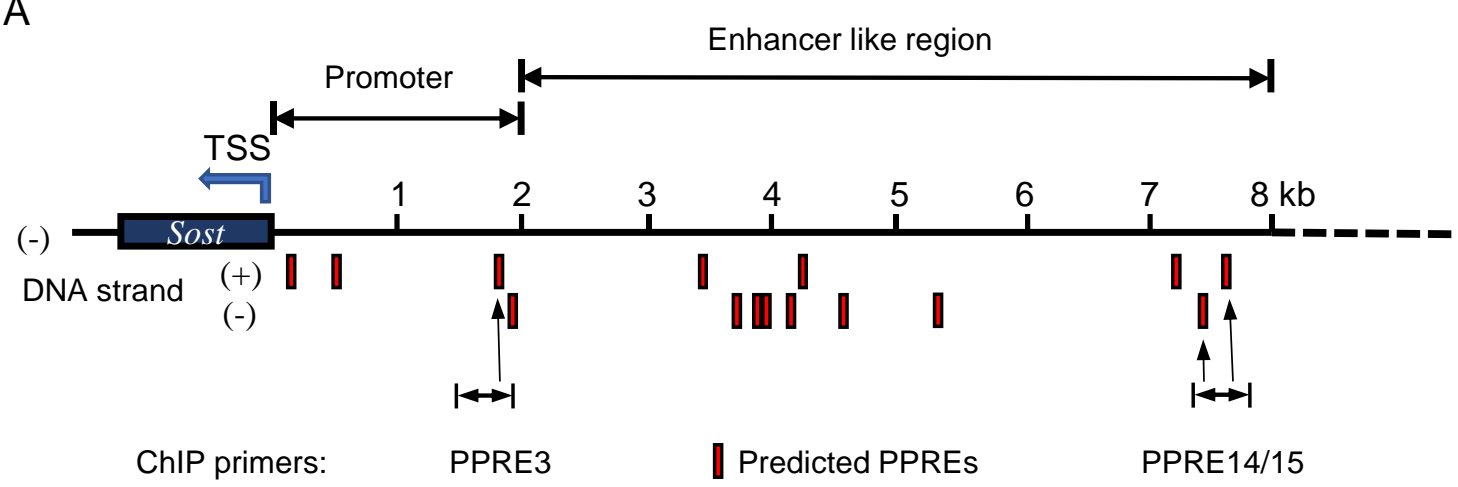

B
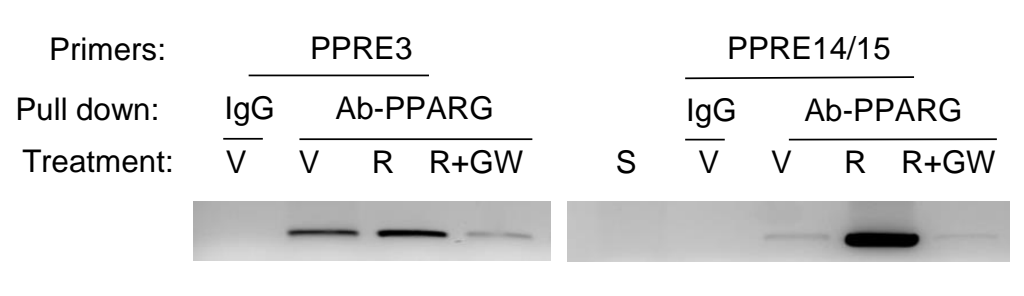

C

Sost

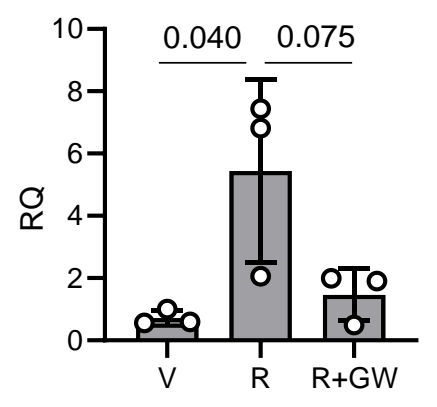

$\mathrm{D}$

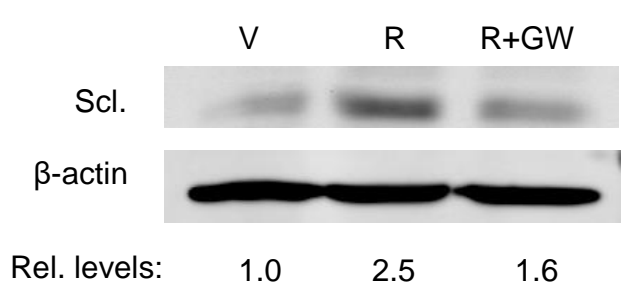

Figure 3. PPARG positively regulates sclerostin expression. A. Schematic positioning of JASPAR projected PPARG response elements (PPREs) upstream of sclerostin transcription start site (TSS). B. Chromatin immunoprecipitation (ChIP) assay end point agarose gel image (full image of the gel is presented in Suppl. Fig. 1). ChIP was performed on osteocyte-like MLO-Y4 cells targeting PPRE3 and PPRE14/15. Cells were treated for $24 \mathrm{~h}$ with either vehicle $(\mathrm{V})$, or $1 \mu \mathrm{M}$ rosiglitazone $(\mathrm{R})$ or combination of $1 \mu \mathrm{M}$ R and $10 \mu \mathrm{M}$ GW9662 antagonist (R+GW) and ChIP assays were performed, as described in Material and Methods. S - sonicated lysate (no antibody pulldown). C. Expression of Sost mRNA in MLO-Y4 cells treated for 3 days with either vehicle (V), or rosiglitazone (R), or combination of rosiglitazone and GW9662 (R+GW) at the same doses as in (B). One-way ANOVA followed by Tukey's post hoc analysis was performed for significance calculation. D. Western blot of sclerostin protein levels in MLO-Y4 cells treated as in (C). Sclerostin protein levels were normalized to $\beta$-actin levels measured as band density using Image $\mathbf{J}$ and relative levels of expression had been calculated and shown below Western blot images. 
The pattern of dynamic changes of PPARG interactions with PPREs was paralleled by regulation of Sost mRNA expression in the same MLO-Y4 cells. Treatment with rosiglitazone upregulated Sost expression, which in the presence of GW9662 antagonist was attenuated (Fig. 3C). This was followed by changes in the sclerostin protein levels. Treatment with rosiglitazone increased sclerostin by 2.5-fold, which was then reduced to 1.6-fold in the presence of GW9662 antagonist (Fig. 3D). These results demonstrate that PPARG is a positive regulator of Sost transcription and sclerostin protein production. Most importantly, the PPARG transcriptional activity regulating sclerostin can be modulated pharmacologically.

\section{Expression of WNT signaling and bone formation markers is elevated in endosteal osteoblasts of $\gamma_{\mathrm{OT}}^{\mathrm{KO}}$ mice, as compared to controls}

Sclerostin acts as an inhibitor of WNT and BMP signaling in osteoblasts. Consistent with low levels of sclerostin in $\gamma \mathrm{OT}^{\mathrm{KO}}$ osteocytes, the expression of members of WNT signaling is upregulated in endosteal osteoblasts. Osteoblasts isolated from $\gamma \mathrm{OT}^{\mathrm{KO}}$ mice have increased expression of WNT ligands, Wnt10b and Wnt16, and $\beta$-catenin, the transcriptional mediator of canonical WNT pathway activity (Fig. 4A). This is followed by increased expression of genes, which are positively regulated by this signaling, including Axin2, Connexin43 and Cyclin D (Fig. 4B). We have also analyzed expression of osteoblast-specific BMPs, Bmp2 and Bmp4, and found that Bmp4 expression is significantly increased in endosteal osteoblasts derived from $\gamma \mathrm{OT}^{\mathrm{KO}}$ mice (Fig. 4C). Finally, an increase in activity of WNT and BMP pathways is reflected in upregulation of osteoblast-specific transcription factors (Runx2 and Dlx5) and alkaline phosphatase (Alp), a regulator of bone matrix mineralization (Fig. 4D). 
A

Wnt10b

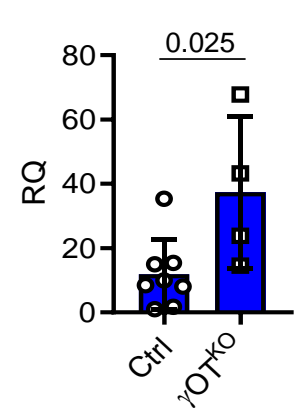

B

$$
\text { Cyclin D }
$$

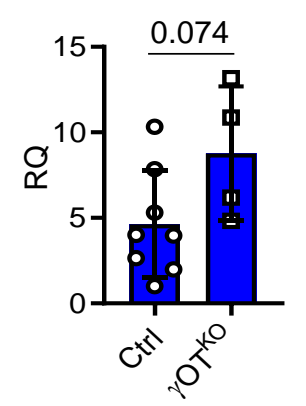

C

Bmp2

Bmp4
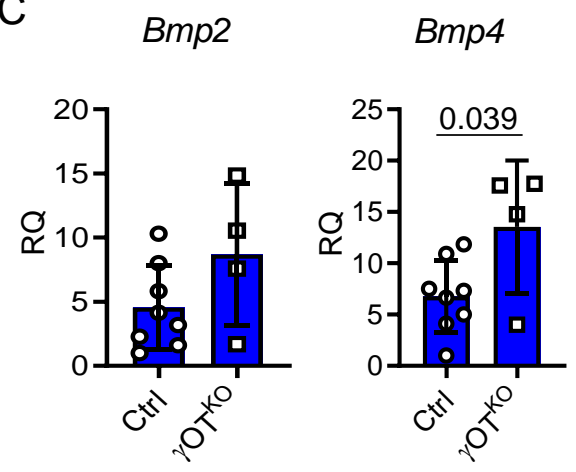

Wnt16

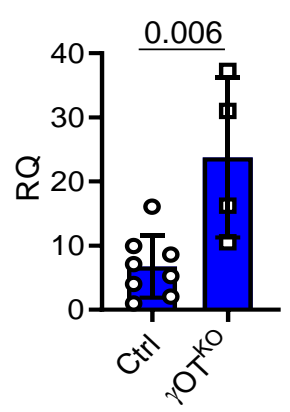

Axin2

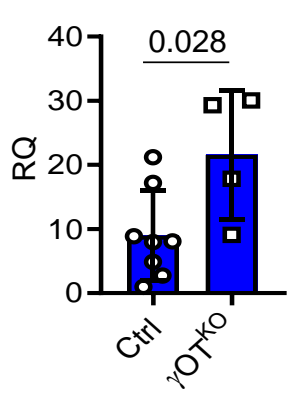

$\beta$-catenin

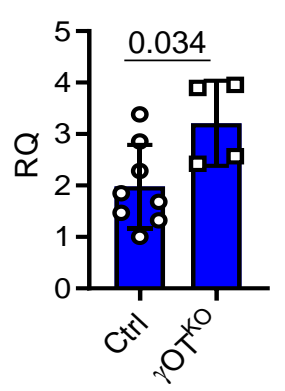

Connexin43

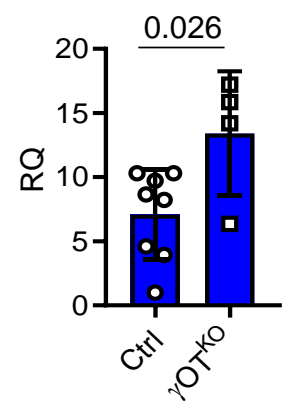

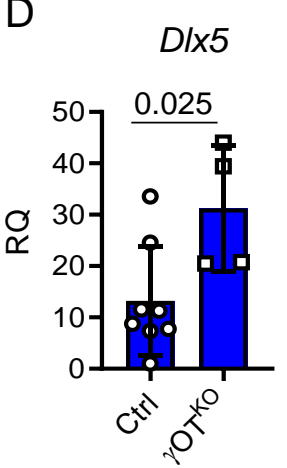
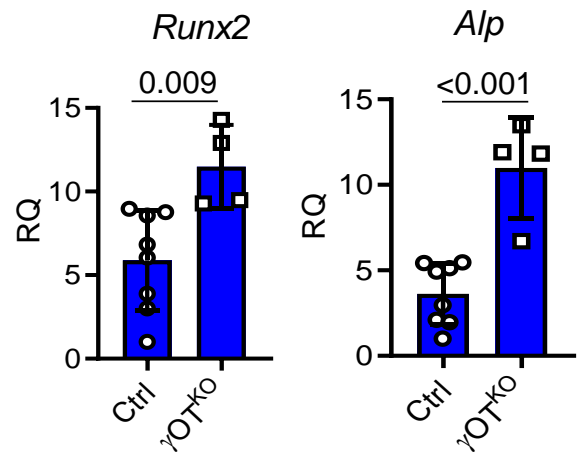

Figure 4. Relative expression of signaling pathways and osteoblast gene markers analyzed in fraction of endosteal osteoblasts freshly isolated by differential collagen digestion of femora cortical bone of 6.5 mo old males. A. WNT pathway signaling gene markers. B. Expression of genes positively regulated by WNT signaling. C. BMP signaling gene markers. D. Expression of genes positively regulated by WNT and BMP signaling and representing markers of bone forming osteoblasts. $\mathrm{Ctrl}(\mathrm{n}=8)$ and $\gamma_{\mathrm{OT}}{ }^{\mathrm{KO}}(\mathrm{n}=4)$. Statistical significance was calculated using parametric unpaired Student's t test.

\section{$\mathrm{OT}^{\mathrm{KO}}$ mice have higher BMD and increased trabecular, but not cortical, bone mass}

In support of increased WNT signaling and osteoblast activity, $\gamma \mathrm{OT}^{\mathrm{KO}}$ mice, both males and females, have high bone mass. In males, an increased global bone mineral density (BMD) is 
noticed as early as 4 mo of age, while in females it develops later in life (Fig. 5A). An analysis of bone formation rate in calcein double-labeled trabecular bone of proximal tibia showed that both $\gamma \mathrm{OT}^{\mathrm{KO}}$ males and females at the age of 6 mo have higher bone formation rate (BFR) as compared to Ctrl mice with intact PPARG in osteocytes (Fig. 5B).
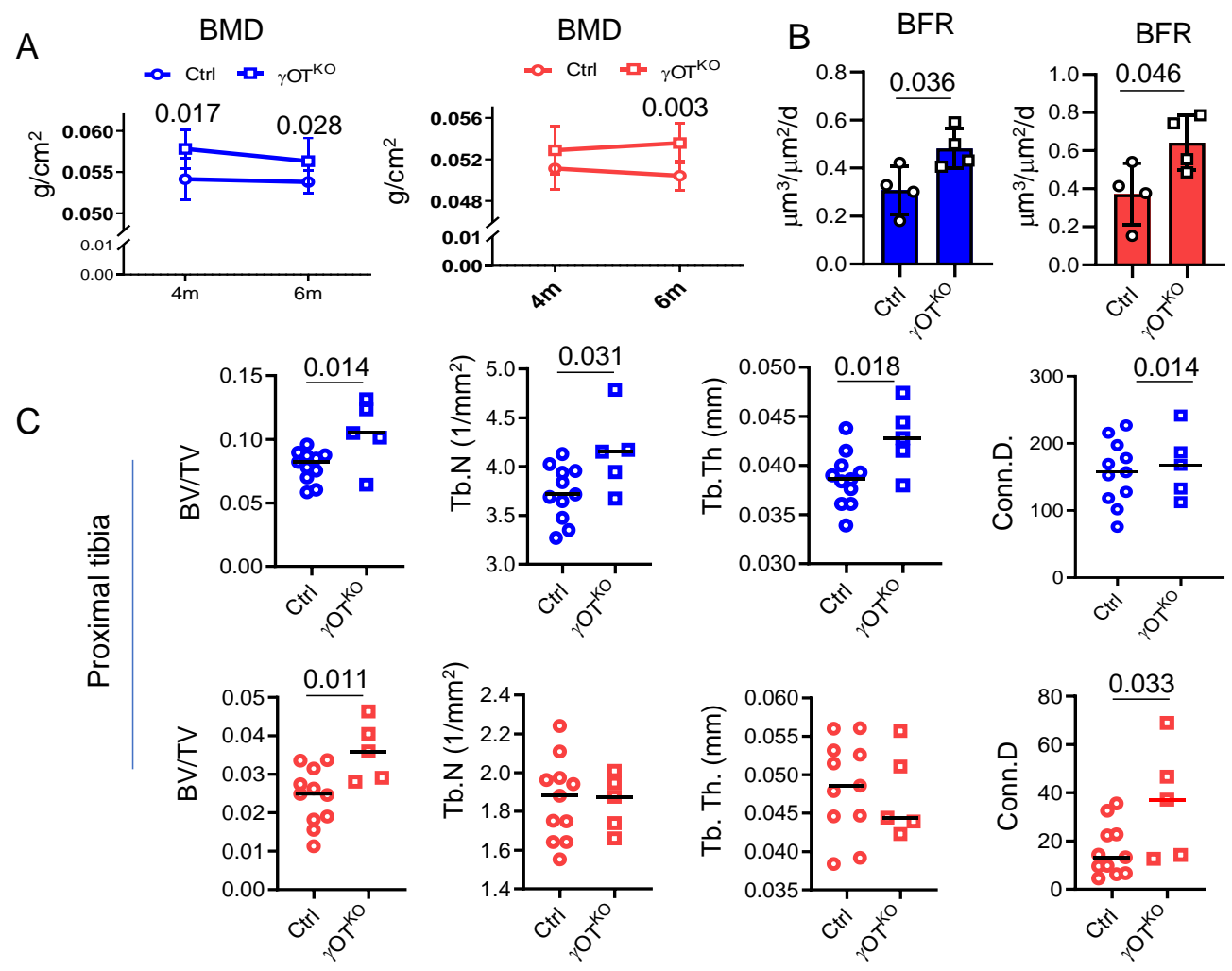

D
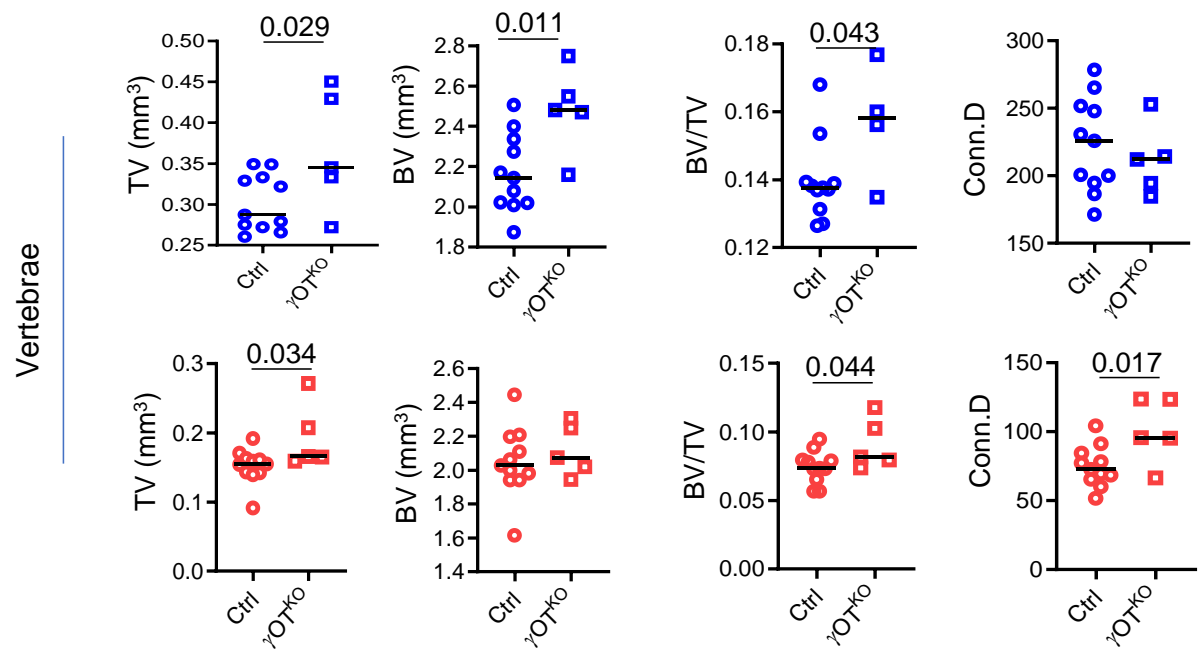
Figure 5. Both males (blue) and females (red) $\gamma_{\mathrm{OT}}^{\mathrm{KO}}$ mice exhibit high bone mass and high bone formation. A. Measurements of global BMD using DXA at 4 mo and 6 mo of age. $\mathrm{Ctrl}(\mathrm{n}=11)$ and $\gamma \mathrm{OT}^{\mathrm{KO}}(\mathrm{n}=5)$. B. Bone formation rate (BFR) in 6.5 mo old males and 7 mo old females measured in calcein double-labeled tibia. $\mathrm{Ctrl}(\mathrm{n}=4)$ and $\gamma \mathrm{OT}{ }^{\mathrm{KO}}(\mathrm{n}=4)$. C. $\mu \mathrm{CT}$ measurements of trabecular bone in proximal tibia. D. $\mu$ CT measurements of trabecular bone in L4 vertebrae. C and D. Ctrl $(n=11)$ and $\gamma_{\mathrm{OT}}^{\mathrm{KO}}(\mathrm{n}=5)$. TV - tissue volume, BV - bone volume, BV/TV - trabecular bone mass, Tb.N trabecular number, Tb.Th - trabecular thickness, Conn.D - connectivity density. Statistical significance was calculated using parametric unpaired Student's $t$ test.

An analyses of bone microarchitecture using micro-computed tomography (mCT), showed that both sexes have increased trabecular bone mass in appendicular and axial skeleton (Fig. 5C and 5D). In males at 6 mo of age, high trabecular bone mass in proximal tibia was consistent with high trabecular number and thickness, whereas in females at the same age high trabecular bone mass correlated rather with structural changes at the level of connectivity density (Fig. 5C). Similarly, both males and females have high trabecular bone mass in L4 vertebra which correlated with increased connectivity density (Fig. 5D). Interestingly, in both sexes the vertebra body is larger in $\gamma \mathrm{OT}^{\mathrm{KO}}$, as compared to $\mathrm{Ctrl}$ mice (Fig. 5D). Remarkably, the changes in global BMD and trabecular bone mass were not followed by changes in properties of tibia cortical bone. The cortical thickness, bone area, marrow area, and bone material properties did not differ in $\gamma \mathrm{OT}^{\mathrm{KO}}$ and Ctrl regardless of sex (Suppl. Table S2). The skeletal phenotype of $\gamma \mathrm{OT}^{\mathrm{KO}}$ mice is consistent with a phenotype of mice with sclerostin ablated under $10 \mathrm{~kb}$ Dmpl Cre control, which are characterized with high trabecular bone mass and an absence of effect on cortical bone (24).

\section{PPARG in osteocytes regulates marrow fat volume at least in part via sclerostin axes}

Besides regulation of WNT signaling in osteoblasts, sclerostin is also implicated in regulation of marrow adipocyte differentiation, probably through the inhibitory effect on WNT pathway activity in cells of adipocyte lineage (25). In general, marrow fat content in tibia of 
$\gamma \mathrm{OT}^{\mathrm{KO}}$ mice is decreased. In males, the decrease in total volume of bone marrow adipose tissue (BMAT) is mostly due to its decrease in distal part since the proximal tibia contains very little fat

A

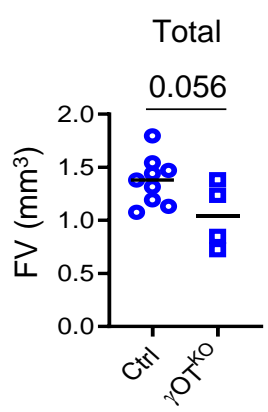

B

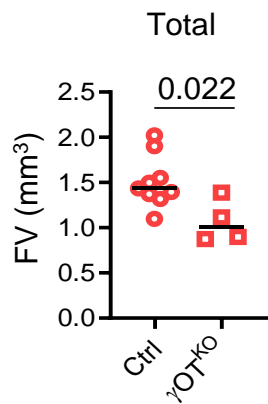

D

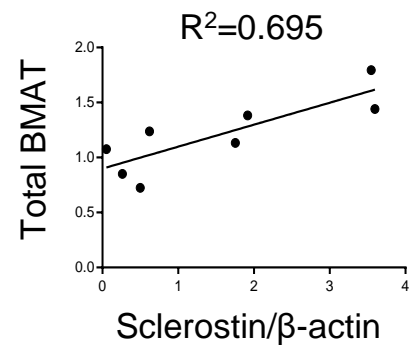

C
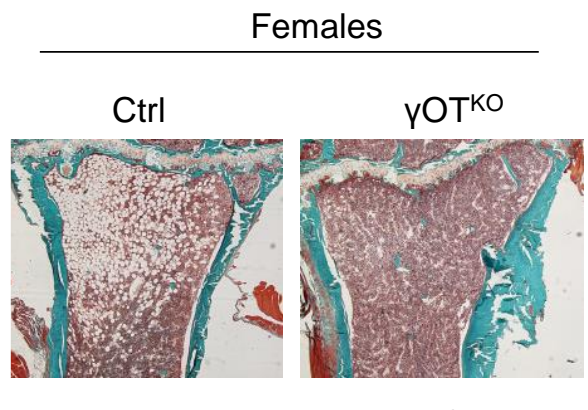

Ctrl

$$
\text { yOTKO }
$$
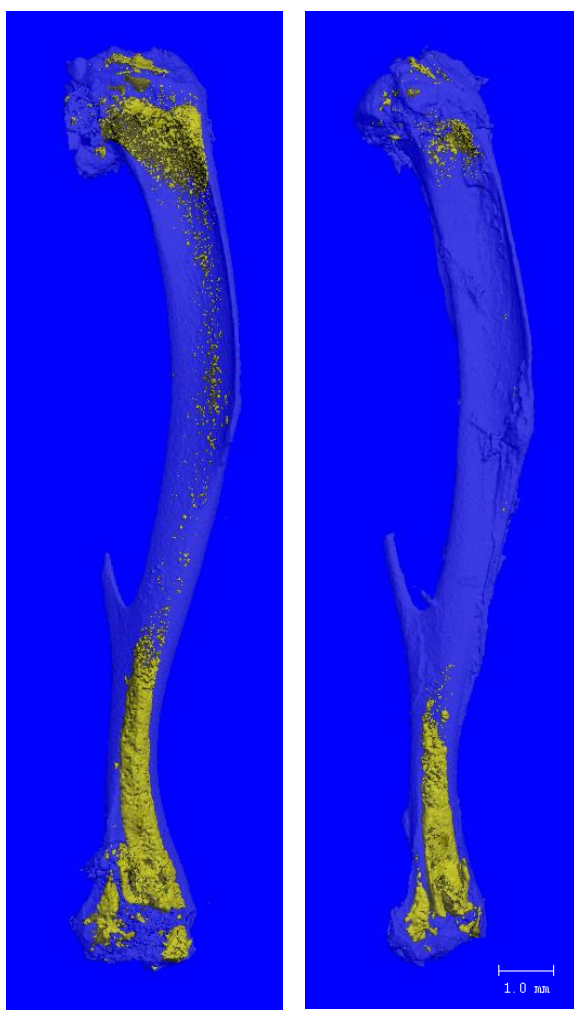

Figure 6. PPARG in osteocytes regulates marrow fat content. A. BMAT content in tibia of 6.5 mo old males $\gamma \mathrm{OT}^{\mathrm{KO}}(\mathrm{n}=4)$ and $\mathrm{Ctrl}(\mathrm{n}=9)$ mice. Measurements were done using $\mu \mathrm{CT}$ after decalcification of tibia followed by $\mathrm{OsO}_{4}$ staining. B. BMAT measurements in tibia of 7 mo old females $\gamma \mathrm{OT}{ }^{\mathrm{KO}}(\mathrm{n}=4)$ and $\mathrm{Ctrl}(\mathrm{n}=9)$ mice. C. Representative Goldner's Trichrome stained proximal tibia of female Ctrl and $\gamma \mathrm{OT}{ }^{\mathrm{KO}}$ mice and renderings of $\mathrm{OsO}_{4}$ stained entire tibia (below) $\mu \mathrm{CT}$ images of female $\mathrm{Ctrl}$ and $\gamma \mathrm{OT}{ }^{\mathrm{KO}}$ mice. Bar on $\mathrm{mCT}$ renderings indicates $1 \mathrm{~mm}$. D. Pearson correlation of marrow fat volume and PPARG protein levels in osteocytes of Ctrl and $\gamma \mathrm{OT}^{\mathrm{KO}}$ mice (males, $\mathrm{n}=8$ ). Statistical significance was calculated using parametric unpaired Student's t test. 
in 6 mo old animals (Fig. 6A). However, in females, which have much more BMAT in proximal tibia than males, PPARG ablation in osteocytes resulted in substantial reduction of BMAT in this location (Fig. 6B and 6C). Similarly to males, females have decreased BMAT volume in distal tibia. Pearson correlational analysis of BMAT volume and osteocyte levels of sclerostin protein in the same animals resulted in the partial but statistically significant coefficient of determination, with the value $\mathrm{R}^{2}=0.695$ (Fig. 6D).

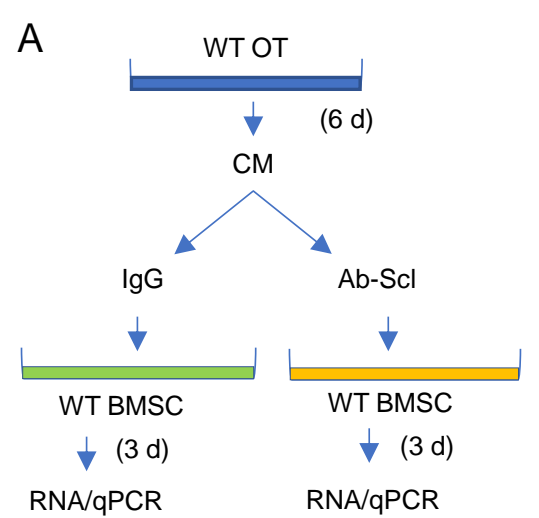

C

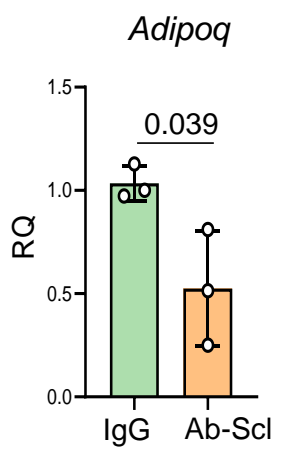

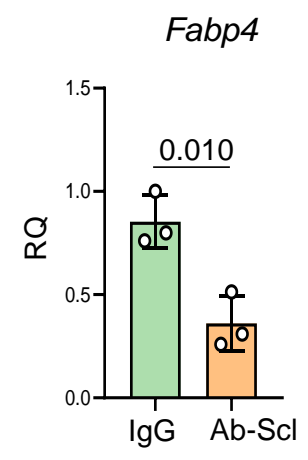
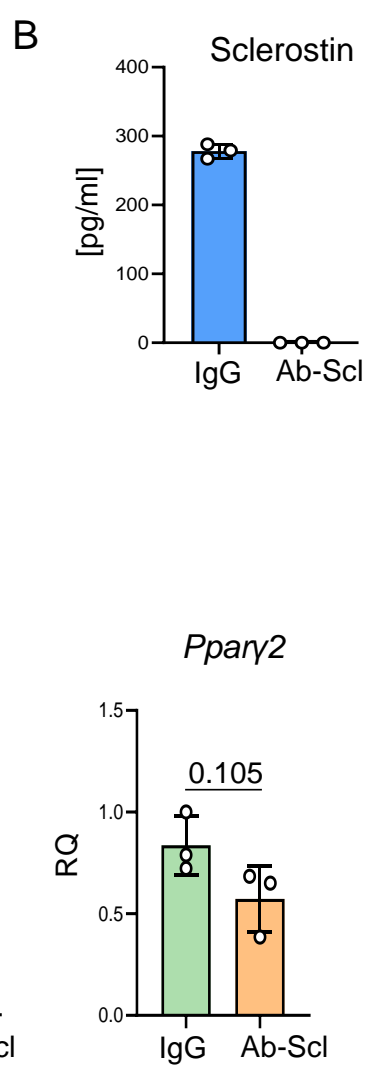

Figure 7. Osteocyte derived sclerostin positively contributes to marrow adipocyte differentiation. A. Schematic showing experimental design of co-culture of BMSC with intact or sclerostin depleted conditioned medium (CM). One group of adherent BMSC culture received IgG depleted CM from primary osteocytes (control group) while the other group received sclerostin depleted CM (group of interest). B. ELISA measurements of sclerostin level in CM after anti-SOST antibody mediated depletion. C. Expression of adipocytic and osteoblastic gene markers in adherent BMSCs treated with CM from primary osteocytes IgG or sclerostin $(\mathrm{Ab}-\mathrm{Scl})$ depleted. Statistical significance was calculated using parametric unpaired Student's t test. 
The coculture experiment assessed sclerostin contribution to the regulation of BMSC lineage allocation toward adipocytes (Fig. 7A). Conditioned media, collected from primary cultures of osteocytes isolated from femora of intact C57BL/6 mice and depleted from sclerostin with specific antibodies (Fig. 7B), decreased expression of Adiponectin and Fabp4, and have a tendency to decrease an expression of adipocyte-specific Ppary2, in recipient BMSC isolated from the same mice (Fig. 7C).

A significant decrease in BMAT volume in $\gamma \mathrm{OT}^{\mathrm{KO}}$ mice, together with a partial correlation between sclerostin levels and BMAT volume, and a decrease in adipocytic phenotype of BMSC subjected to CM depleted from sclerostin, indicate that PPARG in osteocytes is essential for physiological BMAT formation and that sclerostin contributes to this process.

\section{$\gamma \mathrm{OT}^{\mathrm{KO}}$ mice are partially resistant to rosiglitazone-induced bone loss}

The evidence presented in Fig. 1C and Fig. 3, collectively imply that PPARG activated with rosiglitazone increases sclerostin production. It has been documented extensively by our lab and others that rosiglitazone decreases bone mass in mice and humans. Therefore, we asked a question of a contribution of osteocyte PPARG to the skeletal effects of rosiglitazone. Female mice at the age of 10 mo were fed a diet supplemented with rosiglitazone for 4 weeks. As shown in Fig. 8A, a global bone mineral density (BMD) decreased substantially in Ctrl mice as a result of treatment, while BMD of $\gamma \mathrm{OT}^{\mathrm{KO}}$ mice did not change. However, mCT examination of trabecular bone mass in vertebra body showed a tendency to decrease in $\gamma \mathrm{OT}^{\mathrm{KO}}$ mice. While $\mathrm{BV} / \mathrm{TV}$ of Ctrl mice decreased significantly by $34.4 \%$, the vertebra BV/TV of $\gamma \mathrm{OT}{ }^{\mathrm{KO}}$ mice decreased by $20.7 \%$ although did not achieve statistical significance (Fig. 8B). More detailed examination of trabecular microarchitecture confirmed that PPARG in osteocytes contributes to a great extend to the bone loss due to rosiglitazone treatment. While in Ctrl mice rosiglitazone 
significantly decreased trabeculae number and increased spacing between trabeculae, the $\gamma \mathrm{OT}{ }^{\mathrm{KO}}$ mice are largely protected from these effects, although some tendency to changes in these

A

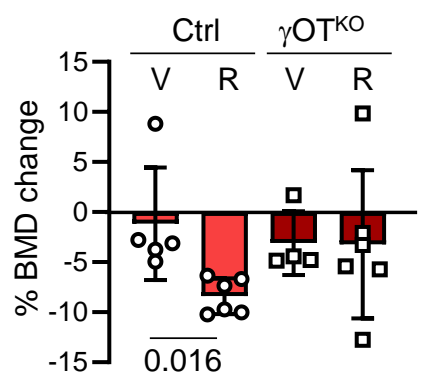

B

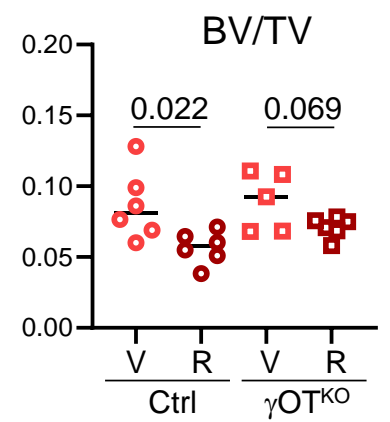

Tb.N

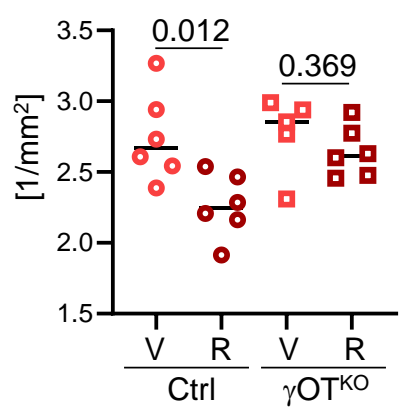

Tb.Th

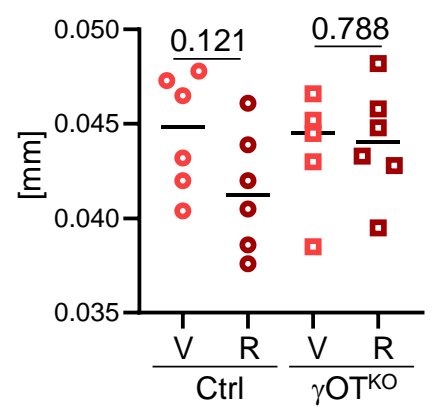

Tb.Sp

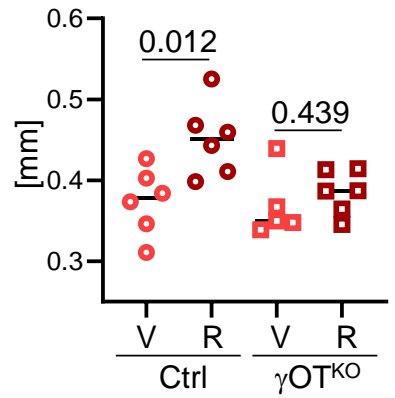

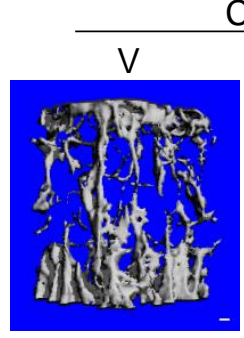

Ctrl

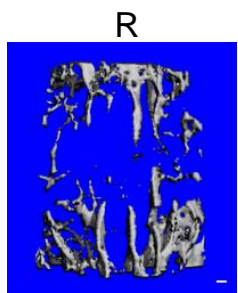
$\gamma \mathrm{OTKO}$

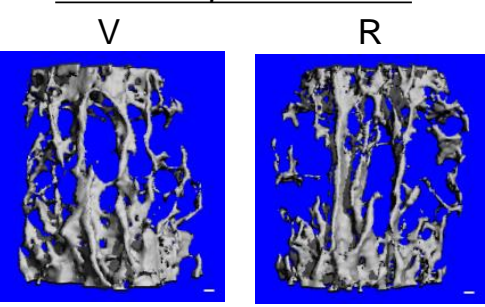

C
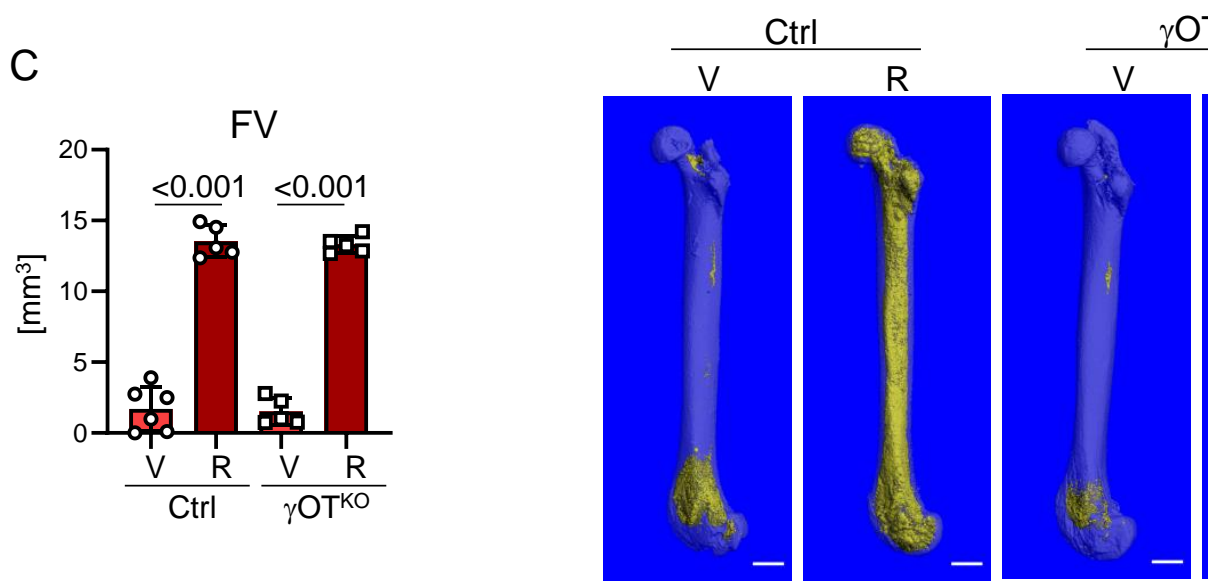

OTKO
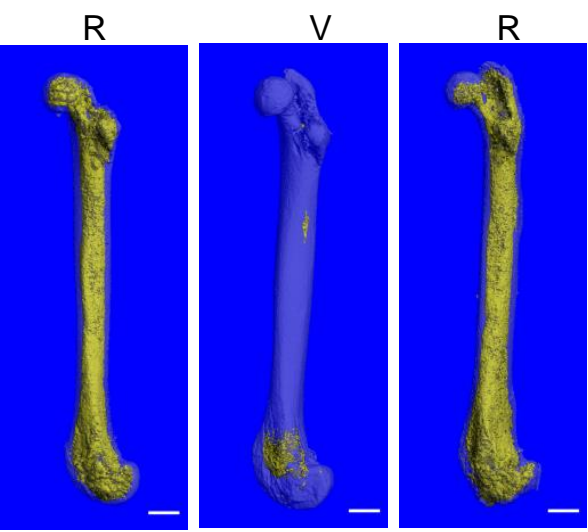
Figure 8. Bone of $\gamma \mathrm{OT}^{\mathrm{KO}}$ mice is partially protected from the detrimental effects of rosiglitazone. A. Percent change in global BMD after 4 wks treatment of 10 mo old females with rosiglitazone (25 $\mathrm{mg} / \mathrm{kg} / \mathrm{d}$ ). n=4-6/group. B. $\mu \mathrm{CT}$ measurements of trabecular bone in L4 vertebrae and representative renderings. n=5-6/group. BV/TV - trabecular bone mass, Tb.N - trabecular number, Tb. $\mathrm{Th}-$ trabecular thickness, Tb.Sp - trabecular spacing. Bar on renderings indicates $0.1 \mathrm{~mm}$. C. Left - Total fat volume in femora measured with $\mu \mathrm{CT}$ after decalcification and staining with $\mathrm{OsO}_{4}$ ( $\mathrm{n}=5$ /group). Right - Representative mCT renderings. Bar on renderings indicates $1 \mathrm{~mm}$. Significant $\mathrm{p}$ values are indicated over horizontal lines, statistical significance of rosiglitazone effect was calculated using parametric unpaired Student's t test.

parameters maybe observed. An analysis of trabecular bone in distal femur was unsuccessful due to the very low trabeculae bone fraction (approx. 1\%) left in this location in Ctrl older female mice and we did not detect significant changes in femora cortical bone which had been previously reported in male mice (26).

Surprisingly, PPARG deficiency in osteocytes did not protect from accumulation of large quantities of marrow fat in response to rosiglitazone treatment (Fig. 8C). These results indicate that TZDs-related bone loss rely on PPARG function in osteocytes and may include sclerostin regulation, while proadipocytic effect of TZDs is separate and probably consists of direct effect on BMSC, which in $\gamma \mathrm{OT}^{\mathrm{KO}}$ model have unaffected expression of PPARG protein. This also means that PPARG in osteocytes does not play a significant role in rosiglitazone-induced BMAT accumulation.

\section{Discussion}

Presented studies provide compelling evidence that PPARG in osteocytes plays an important role in transcriptional regulation of sclerostin expression. First, we have shown that there is an excellent positive correlation between protein levels of PPARG and sclerostin in osteocytes. It is notable that in the absence of PPARG there is an absence of sclerostin, which is pointing to this nuclear receptor as essential for sclerostin production. Consequently, PPARG 
activation with full agonist rosiglitazone increases Sost transcript expression and sclerostin protein levels. Second, we have identified multiple PPRE sequences upstream of transcription start site, and demonstrated that at least two of them bind PPARG protein with dynamics reflecting status of PPARG activation. Thus, PPARG on molecular levels actively interacts with Sost gene DNA regulatory elements located in promoter/enhancer fragment upstream of TSS. Third, an ablation of PPARG in osteocytes leads to increased bone mass and decreased physiological levels of BMAT. Increases in the global BMD and trabecular bone mass, which are associated with increased WNT signaling in endosteal osteoblasts, are expected outcome of sclerostin deficiency in osteocytes $(13,24)$. Finally, PPARG deficiency in osteocytes mitigates rosiglitazone-induced bone loss to a large degree, but does not prevent massive accumulation of fat in marrow cavity, indicating that osteocytes are the major contributors to the TZD-induced bone loss, but not to the medically-induced marrow adiposity. Although, we cannot conclude with certainty that the skeletal resistance to rosiglitazone treatment is entirely mediated by a lack of sclerostin, however presented results provide proof of concept that pharmacologic modulators of PPARG activity reach osteocytes in their lacunae and exert the function including differential regulation of sclerostin protein.

Finding the functional link between PPARG and sclerostin is of great importance, because both proteins are pharmacologic targets for existing therapies to treat diabetes and osteoporosis, respectively. This finding provides means to explore therapeutic overlap between anti-diabetic and anti-osteoporotic therapies. In fact, our previous study showed that anabolic effect on bone of a novel type of insulin sensitizers, which act as inverse agonists in respect to the PPARG pro-adipocytic and anti-osteoblastic activity, was associated with down-regulation of Sost expression in osteocytes (12). Here, we have demonstrated that PPARG is a positive 
regulator of sclerostin protein expression by showing that its ablation from osteocytes shuts down, while its activation increases, sclerostin production.

A potential prospect to pharmacologic targeting PPARG to reduce sclerostin in bone have several caveats which need to be corroborated. Does PPARG regulate sclerostin in other organs too, including aorta, kidney or joints? These are potential off-targets for therapies with sclerostin antibodies (14-18,20,22). Interestingly, we have shown that deletion of PPARG followed by an absence of sclerostin in osteocytes does not dramatically change the levels of sclerostin in circulation. This corresponds to the observation of others that there is a weak correlation between the levels of sclerostin production in osteocytes and the levels in circulation, indicating a substantial contribution of other organs to the total sclerostin levels $(23,24,27)$. Thus, paracrine effect of sclerostin produced in osteocytes directly affects endosteal osteoblasts, with a little, if any, contribution of circulating sclerostin. Our results are supporting this notion. The lack of sclerostin production in osteocytes results in increased bone mass and activation of endosteal osteoblasts, even in the presence of relatively unchanged sclerostin levels in circulation. However, it needs to be addressed whether regulation of sclerostin expression in other organs is also under the control of PPARG. An existence of multiple PPRE sequences in the Sost promoter region may indicate tissue-specific regulation, and may hold a premise for differential modulation of PPARG activities controlling sclerostin production specifically in osteocyte.

Since discovery of sclerostin and its encoding gene Sost, there have been great efforts to identify bone-specific transcriptional mechanisms regulating its expression. The transcriptional control of Sost is complex and implicates number of signaling pathways and only few identified transcription factors, which directly bind to the promoter region and regulate its activity. Several signaling pathways have been recognized as important for Sost expression, the activities of 
which converging on either the promoter region or enhancer elements of Sost gene. Among them, the most prominent is PTH signaling, which acts on ECR5 enhancer via MEF2c/HDAC5 transcriptional regulators, and negatively regulates Sost expression in osteocytes (28-30). However, there is ample of evidence for other signaling mechanisms acting either in a positive (BMP, TNF $\alpha$, Vitamin D, IL-1 $\alpha$, IL-1 $\beta$, TWEAK, RA, and calcitonin), or a negative (PGE2, Oncostatin M, Cardiotrophin, and Sirtuin1), or both manners determined by biological context (TGF $\beta$, glucocorticoids, estrogen, and hypoxia), and interacting with either an immediate promoter region or more distal enhancers $(31,32))$.

Mechanistically, activities of these signaling are channeled to the interaction of specific transcriptional regulators with cognate regulatory elements in the Sost promoter/enhancer regions. Up to date, few of them have been identified for their binding to the promoter including RUNX2, OSX/SP7, ZFP467, and TIEG (33-36). An array of loss- and gain-of-function experiments determined that RUNX2, OSX/SP7 and ZFP467 act as positive regulators of Sost promoter activity and that at least between RUNX2 and OSX/SP7, which binding sites are located in the proximal promoter region between -106 bp to -260 bp upstream of TSS, there is a great degree of coordination in this regulation (37). In contrast, TIEG, or TGF $\beta$ inducible early gene-1, has been identified as a negative regulator of sclerostin expression acting downstream of estrogen signaling (36). Interestingly, two identified KLF/SP1 binding sites for TIEG, which are located at -1864 kb and $-1956 \mathrm{~kb}$ upstream of TSS, are in close proximity to the PPRE3 $(-1829 \mathrm{~kb})$, identified here as binding PPARG, and PPRE4 (-1901 kb). It is well recognized that PPARG and estrogen signaling oppose each other during adipose tissue expansion, including BMAT expansion in conditions of estrogen deficiency (38-40). It also has been shown that estrogen is a negative regulator of sclerostin (36). Thus, there is a prospect that PPARG interaction with distal region of Sost 
promoter may be affected by, or may be subjected to, the modulatory effect of neighboring regulatory elements, such as KLF/SP1 perhaps in estrogen dependent manner. On the other hand, two distal PPRE14 and PPRE15, which have very high scores for PPRE consensus sequence and bind PPARG with dymamics coresponding to the status of PPARG activition, are located approximately in the $1 \mathrm{~kb}$ distance upstream to the region identified as enhancer responsive to Vitamin D and possessing a putative VDR binding element (41), which creates a possibility of PPREs interaction with a modulatory element under control of another nuclear receptor, VDR.

On note, we have also identified up to 7 PPREs with relatively good scores, which are clustered within a $1 \mathrm{~kb}$ fragment at the $4 \mathrm{~kb}$ distance from TSS; however, in this study we did not analyze the functional significance of this region to the regulation of Sost expression.

Nevertheless, the pattern of such close clustering is known to facilitating strong interaction with binding proteins, even if the particular consensus sequences are not at the highest scores (42). We have previously identified similar pattern of PPREs distribution in the promoter of genes known to be directly and positively regulated by PPARG including adipocyte-specific gene Fabp4 (Supplementary Fig. 4 in ref. (8)). The significance of this region to Sost gene expression remains to be established for both PPARG basal activity and the activity modulated pharmacologically by either full or inverse agonists. Taking into account the PPRE number, pattern of their distribution, and binding activity of those which were analyzed, it concludes in a strong support for PPARG directly regulating Sost expression via interaction with promoter and enhancer region.

Besides regulating bone remodeling, osteocytes also modulate marrow environment including marrow adiposity with sclerostin having a prominent role in this process. By the fact that sclerostin acts as an inhibitor of WNT pathway activity, it has a role in skewing BMSC 
lineage allocation toward adipocyte and away from osteoblast differentiation. The proadipocytic activity of sclerostin has been reported in several systems of adipocyte differentiation including 3T3-L1 cell line representing progenitors for white adipose tissue (43), ear mesenchymal stem cells, and BMSC (44). Consistent with proadipocytic activity, a decrease in sclerostin protein levels in $\gamma \mathrm{OT}^{\mathrm{KO}}$ mice correlates with a decrease in BMAT volume. However, there are few interesting aspects of this phenotype including different manifestation in respect to sex and skeletal location, and a degree of correlation.

Several research groups including our have shown that BMAT represents heterogenous tissue consisting of adipocytes originating from different progenitors and having different phenotype and function depending on skeletal location and exposure to environmental or hormonal signaling $(40,45,46)$. Here we show that ablation of PPARG in osteocytes affects BMAT in tibia to a different degree in respect to location and animal sex. We observe an approximately $20 \%$ decrease in the volume of distal BMAT regardless of animal sex. However, proximal BMAT in females is affected to much larger degree in $\gamma \mathrm{OT}^{\mathrm{KO}}$ mice indicating of powerful signals derived from osteocytes that regulate BMAT formation. The coculture experiments with intact or sclerostin depleted conditioned media confirm that sclerostin is partially, but not entirely, responsible for this phenotype. The degree of inhibition of adipocyte gene markers in recipient cells in vitro together with a rather modest correlation of BMAT volume in tibia with sclerostin levels in the same bone, suggest that there are other factors under PPARG control in osteocytes, which regulate BMAT specifically in the proximal tibia location in females. We did not observe proximal BMAT phenotype in male tibia, probably because at the age of 6 mo they did not have sufficient amount of BMAT in this location that would allow for non-biased analysis. Nevertheless, the differences in BMAT volume in proximal and distal 
tibia indicate that proximal BMAT is to a large degree dependent on osteocyte signaling under control of PPARG.

The significance of presented study is reinforced by the fact that there is a scarcity of information on the role of PPAR $\gamma$ in osteocytes. To our knowledge, there are only two reports that previously addressed this issue. The first consists of development a model of PPARG deficiency in osteocytes constructed from the same DmplCre driver and Pparyflfl mice, which was characterized with high bone mass including increased cortical bone mass and periosteal bone formation; the phenotype that we did not confirm in our model. This study was limited to male mice and did not focus on the mechanism by which PPARG in osteocytes controls bone mass including regulation of sclerostin expression and bone marrow composition in respect to BMAT (47). The second model, demonstrated that sclerostin expression is increased in osteocyte-like MLO-Y4 in response to treatment with PPARG agonist rosiglitazone, which is consistent with our results; however, the mechanism of this effect was not provided (48).

In presented study, we have focused on the PPARG-sclerostin connection; however, we recognize that this relationship is a part of a larger role which PPARG plays in regulation of osteocyte function supporting bone homeostasis and energy balance; the role which is pursued in another ongoing study. The current study indicates that PPARG is an essential regulator of osteocyte function with substantial fraction of these activities being channeled through sclerostin activities.

\section{Materials and Methods}

\section{Animals}


Osteocyte-specific PPARG knock-out mice, $\gamma \mathrm{OT}^{\mathrm{KO}}$, were developed at the University of Toledo according to the Institutional Animal Care and Utilization Committee protocol. The $\gamma \mathrm{OT}^{\mathrm{KO}}$ mice were constructed by crossing the $10 \mathrm{~kb}$ Dmpl ${ }^{\mathrm{Cre}}$ mice (B6N.FVB-Tg (Dmplcre)1Jqfe/BwdJ) and Ppary ${ }^{\mathrm{fl} / \mathrm{fl}}$ mice (B6.129-Pparg $\left.{ }^{\mathrm{tm} 2 \mathrm{Rev}} / \mathrm{J}\right)$. Both mouse strains have C57BL/6 background and were obtained from the Jackson Laboratory (Bar Harbor, ME). Ppary ${ }^{\mathrm{fl} / f 1}$ mice have loxP sites flanking exons 1 and 2 of Ppary gene. The Cre-recombinase is under late osteoblast/osteocyte specific10kb $\mathrm{Dmpl}$ promoter. The desired osteocyte-specific $\gamma \mathrm{OT}^{\mathrm{KO}}$ and

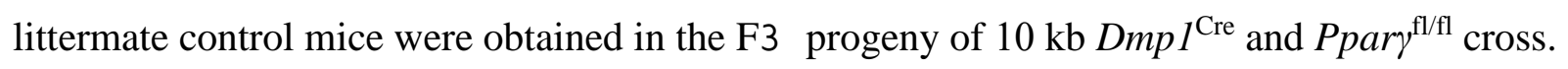
As controls, mice with either $D m p 1^{\text {Cre }}$ only and/or Ppary ${ }^{\mathrm{fl} / \mathrm{fl}}$ only genotypes were used. Animals were housed under $12 \mathrm{~h}$ dark-light cycle with unlimited access to chow (Teklad global $16 \%$ protein rodent diet; code 2916) and water. During breeding of these mice, breeding diet (Teklad global 19\% protein extruded rodent diet; code 2919) was used.

For genotyping, tissue samples were collected at weaning using tail clipping and genomic DNA isolated after proteinase K (Sigma-Aldrich, Cat\#P5568) digestion. PCR primers published by the Jackson Laboratory were used for genotyping. The Cre recombinase insertion was detected with following primers: F - TTG CCT TTC TCT CCA CAG GT and R - CAT GTC CAT CAG GTT CTT GC); while to differentiate between Ppary $\gamma^{\mathrm{fl} / \mathrm{fl}}$ (homozygous), Ppar $\gamma^{\mathrm{fl} /+}$ (heterozygous) and $\mathrm{Ppar}^{+/+}$(wildtype) strains following primers were used: F TGGCTTCCAGTGCATAAGTT and R - TGTAATGGAAGGGCAAAAGG. Taq polymerase (Go Taq; Promega, Cat\#M3005) based amplification was used for PCR followed by 1\% agarose gel run for amplicon detection (167 bp for Cre; 200 bp for Ppar $\gamma$ wildtype, 230 bp for Ppar $\gamma$ homozygotes, both $200 \mathrm{bp}$ and 230 bp for Ppary heterozygotes). 
Rosiglitazone supplemented diet experiment: Nine month old female mice (Ctrl and $\gamma \mathrm{OT}^{\mathrm{KO}}$ ) were fed with either regular or rosiglitazone supplemented at the dose $25 \mathrm{mg} / \mathrm{kg} / \mathrm{day}$ diet, as described (26). DXA was performed at the beginning of the study and after 4 weeks of treatment to measure changes in BMD. After 6 weeks of treatment, animals were sacrificed, and tissues harvested. Right femur and vertebrae were scanned with mCT for bone parameters, then femur was decalcified, stained with $\mathrm{OsO}_{4}$ and scanned for marrow fat.

\section{$D X A$}

Dual Energy X-ray absorptiometry (DXA) was used to measure BMD values longitudinally as the mice age. Measurements were done using Lunar PIXImus II instrument (GE Lunar Corp., Madison, WI) at 4 mo and 6 mo of age and analyzed using LUNAR PIXImus v. 2.10 software provided by manufacturer. Head and metal tag region were excluded from region of interest (ROI).

\section{mCT analysis of bone and marrow fat}

Right tibiae and L4 vertebrae were collected from euthanized mice and stored in 10\% buffered formalin until scanned using $\mu$ CT35 system (Scanco Medical AG, Basserdorf, Switzerland). A minimum $48 \mathrm{~h}$ wait period was maintained before scanning to attain fixation plateau for all bones. mCT scans were performed at 70 peak kilovoltage $(\mathrm{kVp})$ energy and 114$\mu \mathrm{A}$ intensity settings and using $7-\mu \mathrm{m}$ voxel. For proximal tibiae trabecular bone, integration time was $300 \mathrm{~ms}$ and 200 slices were analyzed, for midshaft cortical bone, integration time was 100 ms and 57 slices were analyzed, while for vertebrae, integration time was $100 \mathrm{~ms}$ and 100 slices were analyzed, as recommended (49). 
To visualize marrow lipids, bones were decalcified using Formical 4 (Medline; Cat\# STLB12145) and stained with freshly prepared 2\% aqueous solution of Osmium Tetroxide (Electron Microscopy Sciences, Cat\#19150) in 0.1 M sodium cacodylate buffer (pH 7.4), as described (50). Staining was carried out in an exhaust hood and away from light due to osmium tetroxide toxicity and light sensitivity. Images of lipid depositions were acquired at 70-kVP and $114-\mu \mathrm{A}$ settings and $12-\mu \mathrm{m}$ nominal resolution. Image segmentation was done under global threshold conditions by applying a gray scale threshold of 480-1000 using the per mille scale with the three-dimensional noise filter set to sigma 1.2 and support 2.0. Lipid volumes were calculated directly from individual voxel volumes in three-dimensional reconstructions.

\section{Dynamic histomorphometry}

Sterile filtered Calcein (Sigma, Cat\#C0875-5G) solution $(2.5 \mathrm{mg} / \mathrm{ml})$ prepared in isotonic $2 \% \mathrm{NaHCO}_{3}$ was administered intraperitonially $(20 \mathrm{mg} / \mathrm{kg}$ body weight) to mice, 10 days and 2 days before euthanasia. Left tibia was collected in $70 \% \mathrm{EtOH}$ and embedded in methyl methacrylate (Indiana University Histology Core), and processed for slide preparation and staining. From the slides, images were taken using Nikon Eclipse 80i, for the proximal tibia region adjacent to growth plate. From the images, single and double calcein labelled surfaces were measured using Nikon NIS-Elements BR3.0 in a blinded manner by single operator. Endoosteal bone surface in proximal tibia excluding growth plate surface was considered for measurement. Mineralizing surface (MS/BS), Mineral apposition rate (MAR), Bone formation rate (BFR) were calculated, as recommended (51).

\section{Organ culture conditioned medium experiment}


Conditioned media were collected from organ cultures of femora bone. Femora from six C57BL6 mice were collected, bone marrow was spun out and discarded, the outer surface of bone was cleaned off any remaining muscle fiber and periosteum, and one collagenase digestion followed by one EDTA digestion was performed, as described in (52). Using sterile bone-cutting scissors, the bones were then chopped into 1-2 mm fragments and distributed evenly on collagen coated 6-well plates with $3 \mathrm{ml}$ culture medium/well ( $\alpha$ MEM supplemented with $15 \%$ FBS and $10 \mathrm{mM}$ glucose). Bone pieces were cultured for 6 days at $37 \mathrm{C}$ with $5 \% \mathrm{CO}_{2}$ before conditioned medium (CM) was collected and pooled from all six bone organ cultures. Sclerostin was depleted from one half of the collected osteocyte CM by overnight incubation with antisclerostin antibody (Anti mouse sclerostin Ab, polyclonal Affinity purified; R \& D Systems; cat\# AF1589) in the ratio $20 \mu \mathrm{l}$ antibody per $9 \mathrm{ml} \mathrm{CM}$, and pulled down with Protein A agarose beads (Invitrogen, cat\#15918014). The other half of the conditioned media was subjected to IgG pulldown step (2 $\mu 1$ for $9 \mathrm{ml} \mathrm{CM}$ ) (Anti-mouse IgG monoclonal Ab; Sigma-Aldrich; Cat\#M9144) which was used as a negative control for this assay. Sclerostin levels after depletion were quantified using SOST ELISA kit (Quantikine ELISA, R\&D Systems, Cat\#MSST00).

Recipient BMSC cultures were established from bone marrow isolated from femora of six C57BL6 mice, pooled and plated on 6-well plated at the density $2 \times 10^{6}$ cells/well. After 7 days of growth, the culture medium was then replaced with osteocyte CM either depleted of Sclerostin or IgG in triplicates, and cultured for three more days before processing for RNA isolation followed by analysis of adipocytic and osteoblastic markers using qPCR.

\section{Isolation of osteocyte-enriched and endosteal osteoblast-enriched cell fractions from femora}

An osteocyte-enriched fraction and endosteal osteoblast enriched fraction after sequential collagenase digestion of femora $(52,53)$. Briefly, femora were cleaned off muscles and bone 
marrow was spin-out after removal of epiphysis. The diaphysis-outer-surface was scrapped to remove periosteum and any remaining muscle fibers. Bones were then subjected to alternating digestion with collagenase and EDTA to retrieve endosteal osteoblasts and osteocytes. First two collagenase and EDTA digestions of femora were discarded. Cells from the $3^{\text {th }}$ digestion were collected and represented an endosteal osteoblast enriched fraction 3 (F3). Alternating digestions continued and cells from the $6^{\text {th }}$ digestion were collected and represented osteocyte enriched fraction 6 (F6). The leftover bone pieces were homogenized and added to F6 osteocyte enriched fraction. Cell-type enrichment of isolated fractions was verified by analyzing levels of osteoblasts (Col1, Ocn) and osteocytes (Dmp1, Sost) gene markers expression (Suppl. Fig. S1).

\section{Gene Expression Analysis}

mRNA was isolated using Trizol (Thermo Fisher Scientific, Cat\# 15596026). Half $\mu \mathrm{g}$ RNA was converted to cDNA using the Verso cDNA synthesis kit (Thermo Fisher Scientific, Cat\#AB1453B). Gene transcripts were detected by quantitative real-time PCR using TrueAmp SYBR Green qPCR SuperMix (Smart Bioscience, Cat\#7501-3) and processed with StepOne Plus System (Applied Biosystems, Carlsbad, CA). Relative gene expression was measured by the comparative $\Delta \Delta \mathrm{CT}$ method using $18 S \mathrm{RNA}$ levels for normalization. Primers were designed using OligoPerfect Designer (Thermo Fisher Scientific) and are listed in the Suppl. Table S3.

\section{Protein analysis using Western blots}

Proteins were isolated from cell fraction enriched in osteocytes using TRIzol, following manufacturer's protocol and quantified using BCA assay ( Pierce BCA protein Assay Kit, ThermoScientific, Cat\#23225). Ten $\mu \mathrm{g}$ of protein was loaded on each lane and resolved on $10 \%$ SDS-PAGE using BioRad system and electrophoretically transferred to Immobilon-FL 
membranes. Membranes were blocked at room temperature for $2 \mathrm{~h}$ in Odyssey blocking buffer (LI-COR, cat\#927-50000). Incubation with primary antibody was performed overnight at $4^{\circ}$ with either mouse monoclonal anti-PPAR $\gamma$, 1:500 dilution, (Santa Cruz Biotechnology, Santa Cruz, CA; cat \# Sc-7273), or goat polyclonal anti-sclerostin, 1:500 dilution, (R\&D System, Minneapolis, MN; cat \#AF1589), or mouse monoclonal $\beta$-actin, 1:10,000 dilution, (SigmaAldrich Life Science, St. Louis, MO; cat \#A1978). After incubation, membranes were washed 3 times in TBST (TBS plus $0.1 \%$ Tween 20), and incubated with appropriate secondary antibody, either infrared anti-mouse (680RD; cat\#925-68072) or infrared anti-goat (800CW; cat\#92532214) (LI-COR Bioscience, Lincoln, NE), at 1:10,000 dilution in TBS for $2 \mathrm{~h}$ at room temperature. Immunoreactivity was visualized by infrared scanning in the Odyssey system (LICOR Biosciences). Image $\mathrm{J}$ (version 1.52a) was used to calculate band intensity from Western blots and band intensity of beta-actin was used to normalize PPARG and sclerostin signals.

\section{In-silico mapping of PPREs in Sost regulatory region using JASPAR}

The $8.85 \mathrm{~kb}$ sequence upstream to Sost gene transcription start site (TSS) was obtained from NCBI Genebank database. This sequence was divided into 3 equal fragments $(2.95 \mathrm{~kb}$ each) and analyzed for mouse PPARG::RXRA heterodimer binding sites using JASPAR (version 2020), a database for curated, non-redundant set of transcription factor binding profiles. Position weight matrix (PWM) for mouse PPARG::RXRA binding site was run with relative profile score threshold set at $80 \%$.

\section{ChIP assay}

Chromatin Immunoprecipitation (ChIP) assay to detect PPARG binding to selected PPRE sequences was performed on osteocyte like MLO-Y4 cells (kind gift from Dr. L. Bonewald). 
Cells were cultured on collagen coated $100 \mathrm{~mm}$ plates using $5 \% \mathrm{FBS}, 5 \% \mathrm{CS}$ and $1 \% \mathrm{P} / \mathrm{S}$ in alpha-MEM medium (at 37C with 5\% $\mathrm{CO}_{2}$ ). Optimal passage time was 4-5 days before each splitting with 1:5 ratio after cultures reached $80 \%$ confluency. In each experiment, cells were treated in duplicates with either DMSO as vehicle, PPARG agonist rosiglitazone $(1 \mu \mathrm{M})$ and a combination of rosiglitazone $(1 \mu \mathrm{M})$ and antagonist GW9662 $(10 \mu \mathrm{M})$. Sonicated only and Immunoglobulin $\mathrm{G}(\mathrm{IgG})$ pull-down samples were used as negative controls.

Chromatin Immunoprecipitation protocol was followed (54). Briefly, after $24 \mathrm{~h}$ treatment cells were fixed using 4\% formaldehyde in PBS, lysed, and DNA was sonicated to fragments size 500 - 1000 bp (6 min total time per sample, 12 cycles of 30 s pulse at $50 \%$ amplitude of Qsonica CL-18 sonicator followed by 90s incubation on ice after each cycle). After sonication, samples were incubated with Protein A agarose beads (Invitrogen, Cat\#15918014) for 4h at 4C to reduce background. The beads were spun down and discarded, and either $\operatorname{IgG}(2 \mu 1 / \mathrm{sample}$; Anti-mouse IgG monoclonal Ab; Sigma-Aldrich; Cat\#M9144) or PPARG antibody (10 $\mu \mathrm{l} /$ sample; Anti-mouse PPAR $\gamma$ monoclonal Ab; Santa Cruz Biotechnology;Cat\#Sc-7273) were added and incubated overnight at $4 \mathrm{C}$ followed by adding fresh protein $\mathrm{A}$ agarose beads (60 $\mu \mathrm{l} /$ sample) and incubated for $4 \mathrm{~h}$ at $4 \mathrm{C}$. After spinning down the beads, antibody bound chromatin-PPARG fragments were eluted using elution buffer (54) followed by crosslink reversal (15h at 65C), Proteinase K digestion (1h at 50C) and purification of pulled-down DNA fragments using phenol-chloroform-isoamyl alcohol (25:24:1) extraction. The Taq polymerase (GoTaq) based PCR was performed with specifically designed primers for analyzed PPREs: PPRE3 F - TCTCCCAAGTCTGGAGCAAT and R -CTGAGGTGCAAAAGGAGGAG; PPRE14 \& 15 F - TACCACGTCTCCCCTGTTTC and R - GGGCCTGTGTTTGCATAGTT. 
The presence of specific amplicons (532 bp for PPRE3 and $634 \mathrm{bp}$ for PPRE14/15) was detected using $1 \%$ agarose gel.

\section{Statistical analysis}

Data are represented as means \pm SD and were analyzed by statistical analysis software GraphPad Prism 8. Parametric, unpaired, two-tailed Student's t-test was used for comparing means of two groups. One-way ANOVA followed by Tuckey's test (as post-hoc analysis) was used to compare multiple groups. Pearson correlation was used to measure correlation between experimental variables. A p-value 0.05 and lower was considered as statistically significant. 


\section{Acknowledgements}

This work was supported by grants from the National Institute of Diabetes, Digestive and Kidney Diseases (NIDDK) (R01 DK105825) to PRG and BLC, and the American Diabetes Association Innovative Basic Science Award (1-19-IBS-029) to BLC.

Authors' roles: SB and BLC contributed to experimental design, data analysis, and data interpretation; SB, PJC, and AC conducted the experiments; SB, BLC, and PRG wrote and edited the manuscript, SB and BLC take responsibility for the integrity of the data analysis. 


\section{References}

1. Dirckx N, Moorer MC, Clemens TL, Riddle RC. The role of osteoblasts in energy homeostasis. Nat Rev Endocrinol. 2019;15(11):651-665.

2. Schwartz AV. Diabetes, bone and glucose-lowering agents: clinical outcomes. Diabetologia. 2017;60(7):1170-1179.

3. Lecka-Czernik B. Diabetes, bone and glucose-lowering agents: basic biology. Diabetologia. 2017;60(7):1163-1169.

4. Ahmadian M, Suh JM, Hah N, Liddle C, Atkins AR, Downes M, Evans RM. PPAR $\gamma$ signaling and metabolism: the good, the bad and the future. Nature medicine. 2013;19(5):557-566.

5. Elrod HA, Sun SY. PPARgamma and Apoptosis in Cancer. PPAR research. 2008;2008:704165.

6. Staels B. PPAR $\gamma$ and atherosclerosis. Current Medical Research and Opinion. 2005;21(sup1):S13-S20.

7. Lecka-Czernik B, Gubrij I, Moerman EJ, Kajkenova O, Lipschitz DA, Manolagas SC, Jilka RL. Inhibition of Osf2/Cbfa1 expression and terminal osteoblast differentiation by PPARgamma2. J Cell Biochem. 1999;74(3):357-371.

8. Shockley KR, Lazarenko OP, Czernik PJ, Rosen CJ, Churchill GA, Lecka-Czernik B. PPARgamma2 nuclear receptor controls multiple regulatory pathways of osteoblast differentiation from marrow mesenchymal stem cells. J Cell Biochem. 2009;106(2):232246.

9. Wan Y, Chong L-W, Evans RM. PPAR- $\gamma$ regulates osteoclastogenesis in mice. Nature Medicine. 2007;13(12):1496-1503.

10. Kahn SE, Zinman B, Lachin JM, Haffner SM, Herman WH, Holman RR, Kravitz BG, Yu D, Heise MA, Aftring RP, Viberti G. Rosiglitazone-associated fractures in type 2 diabetes: an Analysis from A Diabetes Outcome Progression Trial (ADOPT). Diabetes care. 2008;31(5):845-851.

11. Zou W, Rohatgi N, Chen TH, Schilling J, Abu-Amer Y, Teitelbaum SL. PPAR-gamma regulates pharmacological but not physiological or pathological osteoclast formation. Nat Med. 2016;22(11):1203-1205. 
12. Stechschulte LA, Czernik PJ, Rotter ZC, Tausif FN, Corzo CA, Marciano DP, Asteian A, Zheng J, Bruning JB, Kamenecka TM, Rosen CJ, Griffin PR, Lecka-Czernik B. PPARG Post-translational Modifications Regulate Bone Formation and Bone Resorption. EBioMedicine. 2016;10:174-184.

13. Delgado-Calle J, Sato AY, Bellido T. Role and mechanism of action of sclerostin in bone. Bone. 2017;96:29-37.

14. Saag KG, Petersen J, Brandi ML, Karaplis AC, Lorentzon M, Thomas T, Maddox J, Fan M, Meisner PD, Grauer A. Romosozumab or Alendronate for Fracture Prevention in Women with Osteoporosis. N Engl J Med. 2017;377(15):1417-1427.

15. Koos R, Brandenburg V, Mahnken AH, Schneider R, Dohmen G, Autschbach R, Marx N, Kramann R. Sclerostin as a potential novel biomarker for aortic valve calcification: an in-vivo and ex-vivo study. J Heart Valve Dis. 2013;22(3):317-325.

16. Brandenburg VM, Kramann R, Koos R, Kruger T, Schurgers L, Muhlenbruch G, Hubner S, Gladziwa U, Drechsler C, Ketteler M. Relationship between sclerostin and cardiovascular calcification in hemodialysis patients: a cross-sectional study. BMC Nephrol. 2013;14:219.

17. Kawaguchi H. Serious Adverse Events With Romosozumab Use in Japanese Patients: Need for Clear Formulation of Contraindications Worldwide. J Bone Miner Res. 2020;35(5):994-995.

18. Saag KG, Curtis JR, Reid IR. Reply to Serious Adverse Events With Romosozumab Use in Japanese Patients: Need for Clear Formulation of Contraindications Worldwide. $J$ Bone Miner Res. 2020;35(5):996-997.

19. Zhu D, Mackenzie NC, Millan JL, Farquharson C, MacRae VE. The appearance and modulation of osteocyte marker expression during calcification of vascular smooth muscle cells. PLoS One. 2011;6(5):e19595.

20. Krishna SM, Seto SW, Jose RJ, Li J, Morton SK, Biros E, Wang Y, Nsengiyumva V, Lindeman JH, Loots GG, Rush CM, Craig JM, Golledge J. Wnt Signaling Pathway Inhibitor Sclerostin Inhibits Angiotensin II-Induced Aortic Aneurysm and Atherosclerosis. Arterioscler Thromb Vasc Biol. 2017;37(3):553-566.

21. Chang JC, Christiansen BA, Murugesh DK, Sebastian A, Hum NR, Collette NM, Hatsell S, Economides AN, Blanchette CD, Loots GG. SOST/Sclerostin Improves Posttraumatic 
Osteoarthritis and Inhibits MMP2/3 Expression After Injury. J Bone Miner Res. 2018;33(6):1105-1113.

22. Wehmeyer C, Frank S, Beckmann D, Bottcher M, Cromme C, Konig U, Fennen M, Held A, Paruzel P, Hartmann C, Stratis A, Korb-Pap A, Kamradt T, Kramer I, van den Berg W, Kneissel M, Pap T, Dankbar B. Sclerostin inhibition promotes TNF-dependent inflammatory joint destruction. Sci Transl Med. 2016;8(330):330ra335.

23. Weivoda MM, Youssef SJ, Oursler MJ. Sclerostin expression and functions beyond the osteocyte. Bone. 2017;96:45-50.

24. Yee CS, Manilay JO, Chang JC, Hum NR, Murugesh DK, Bajwa J, Mendez ME, Economides AE, Horan DJ, Robling AG, Loots GG. Conditional Deletion of Sost in MSC-Derived Lineages Identifies Specific Cell-Type Contributions to Bone Mass and BCell Development. Journal of bone and mineral research : the official journal of the American Society for Bone and Mineral Research. 2018;33(10):1748-1759.

25. Cawthorn WP, Bree AJ, Yao Y, Du B, Hemati N, Martinez-Santibanez G, Macdougald OA. Wnt6, Wnt10a and Wnt10b inhibit adipogenesis and stimulate osteoblastogenesis through a beta-catenin-dependent mechanism. Bone. 2012;50(2):477-489.

26. Lazarenko OP, Rzonca SO, Hogue WR, Swain FL, Suva LJ, Lecka-Czernik B. Rosiglitazone induces decreases in bone mass and strength that are reminiscent of aged bone. Endocrinology. 2007;148(6):2669-2680.

27. Jastrzebski S, Kalinowski J, Stolina M, Mirza F, Torreggiani E, Kalajzic I, Won HY, Lee SK, Lorenzo J. Changes in bone sclerostin levels in mice after ovariectomy vary independently of changes in serum sclerostin levels. J Bone Miner Res. 2013;28(3):618626.

28. Bellido T, Ali AA, Gubrij I, Plotkin LI, Fu Q, O’Brien CA, Manolagas SC, Jilka RL. Chronic Elevation of Parathyroid Hormone in Mice Reduces Expression of Sclerostin by Osteocytes: A Novel Mechanism for Hormonal Control of Osteoblastogenesis. Endocrinology. 2005;146(11):4577-4583.

29. Leupin O, Kramer I, Collette NM, Loots GG, Natt F, Kneissel M, Keller H. Control of the SOST Bone Enhancer by PTH Using MEF2 Transcription Factors. Journal of Bone and Mineral Research. 2007;22(12):1957-1967. 
30. Wein MN, Spatz J, Nishimori S, Doench J, Root D, Babij P, Nagano K, Baron R, Brooks D, Bouxsein M, Pajevic PD, Kronenberg HM. HDAC5 controls MEF2C-driven sclerostin expression in osteocytes. J Bone Miner Res. 2015;30(3):400-411.

31. St John HC, Hansen SJ, Pike JW. Analysis of SOST expression using large minigenes reveals the $\mathrm{MEF} 2 \mathrm{C}$ binding site in the evolutionarily conserved region (ECR5) enhancer mediates forskolin, but not 1,25-dihydroxyvitamin D3 or TGFbeta1 responsiveness. $J$ Steroid Biochem Mol Biol. 2016;164:277-280.

32. Sebastian A, Loots GG. Transcriptional control of Sost in bone. Bone. 2017;96:76-84.

33. Sevetson B, Taylor S, Pan Y. Cbfa1/RUNX2 directs specific expression of the sclerosteosis gene (SOST). J Biol Chem. 2004;279(14):13849-13858.

34. Yang F, Tang W, So S, de Crombrugghe B, Zhang C. Sclerostin is a direct target of osteoblast-specific transcription factor osterix. Biochem Biophys Res Commun. 2010;400(4):684-688.

35. You L, Chen L, Pan L, Gu WS, Chen JY. Zinc finger protein 467 regulates Wnt signaling by modulating the expression of sclerostin in adipose derived stem cells. Biochem Biophys Res Commun. 2015;456(2):598-604.

36. Subramaniam M, Pitel KS, Bruinsma ES, Monroe DG, Hawse JR. TIEG and estrogen modulate SOST expression in the murine skeleton. J Cell Physiol. 2018;233(4):35403551.

37. Perez-Campo FM, Santurtun A, Garcia-Ibarbia C, Pascual MA, Valero C, Garces C, Sanudo C, Zarrabeitia MT, Riancho JA. Osterix and RUNX2 are Transcriptional Regulators of Sclerostin in Human Bone. Calcif Tissue Int. 2016;99(3):302-309.

38. Newell-Fugate AE. The role of sex steroids in white adipose tissue adipocyte function. Reproduction. 2017;153(4):R133-R149.

39. Syed FA, Oursler MJ, Hefferanm TE, Peterson JM, Riggs BL, Khosla S. Effects of estrogen therapy on bone marrow adipocytes in postmenopausal osteoporotic women. Osteoporos Int. 2008;19(9):1323-1330.

40. Lecka-Czernik B, Stechschulte LA, Czernik PJ, Sherman SB, Huang S, Krings A. Marrow Adipose Tissue: Skeletal Location, Sexual Dimorphism, and Response to Sex Steroid Deficiency. Front Endocrinol (Lausanne). 2017;8:188. 
41. Wijenayaka AR, Yang D, Prideaux M, Ito N, Kogawa M, Anderson PH, Morris HA, Solomon LB, Loots GG, Findlay DM, Atkins GJ. 1 $\alpha, 25$-dihydroxyvitamin D3 stimulates human SOST gene expression and sclerostin secretion. Molecular and Cellular Endocrinology. 2015;413:157-167.

42. Czernik PJ, Peterson CA, Hurlburt BK. Preferential binding of MyoD-E12 versus myogenin-E12 to the murine sarcoma virus enhancer in vitro. J Biol Chem. 1996;271(15):9141-9149.

43. Ukita M, Yamaguchi T, Ohata N, Tamura M. Sclerostin Enhances Adipocyte Differentiation in 3T3-L1 Cells. J Cell Biochem. 2016;117(6):1419-1428.

44. Fairfield H, Falank C, Harris E, Demambro V, McDonald M, Pettitt JA, Mohanty ST, Croucher P, Kramer I, Kneissel M, Rosen CJ, Reagan MR. The skeletal cell-derived molecule sclerostin drives bone marrow adipogenesis. Journal of cellular physiology. 2018;233(2):1156-1167.

45. Scheller EL, Doucette CR, Learman BS, Cawthorn WP, Khandaker S, Schell B, Wu B, Ding SY, Bredella MA, Fazeli PK, Khoury B, Jepsen KJ, Pilch PF, Klibanski A, Rosen CJ, MacDougald OA. Region-specific variation in the properties of skeletal adipocytes reveals regulated and constitutive marrow adipose tissues. Nat Commun. 2015;6:7808.

46. Horowitz MC, Berry R, Holtrup B, Sebo Z, Nelson T, Fretz JA, Lindskog D, Kaplan JL, Ables G, Rodeheffer MS, Rosen CJ. Bone marrow adipocytes. Adipocyte. 2017;6(3):193204.

47. Brun J, Berthou F, Trajkovski M, Maechler P, Foti M, Bonnet N. Bone Regulates Browning and Energy Metabolism Through Mature Osteoblast/Osteocyte PPAR $\gamma$ Expression. Diabetes. 2017;66(10):2541-2554.

48. Mabilleau G, Mieczkowska A, Edmonds ME. Thiazolidinediones induce osteocyte apoptosis and increase sclerostin expression. Diabet Med. 2010;27(8):925-932.

49. Bouxsein ML, Boyd SK, Christiansen BA, Guldberg RE, Jepsen KJ, Muller R. Guidelines for assessment of bone microstructure in rodents using micro-computed tomography. J Bone Miner Res. 2010;25(7):1468-1486.

50. Pai VM, Kozlowski M, Donahue D, Miller E, Xiao X, Chen MY, Yu Z-X, Connelly P, Jeffries $\mathrm{K}$, Wen $\mathrm{H}$. Coronary artery wall imaging in mice using osmium tetroxide and micro-computed tomography (micro-CT). J Anat. 2012;220(5):514-524. 
51. Dempster DW, Compston JE, Drezner MK, Glorieux FH, Kanis JA, Malluche H, Meunier PJ, Ott SM, Recker RR, Parfitt AM. Standardized nomenclature, symbols, and units for bone histomorphometry: a 2012 update of the report of the ASBMR Histomorphometry Nomenclature Committee. J Bone Miner Res. 2013;28(1):2-17.

52. Stern AR, Stern MM, Van Dyke ME, Jähn K, Prideaux M, Bonewald LF. Isolation and culture of primary osteocytes from the long bones of skeletally mature and aged mice. Biotechniques. 2012;52(6):361-373.

53. Kramer I, Halleux C, Keller H, Pegurri M, Gooi JH, Weber PB, Feng JQ, Bonewald LF, Kneissel M. Osteocyte Wnt/beta-catenin signaling is required for normal bone homeostasis. Mol Cell Biol. 2010;30(12):3071-3085.

54. Lee TI, Johnstone SE, Young RA. Chromatin immunoprecipitation and microarray-based analysis of protein location. Nat Protoc. 2006;1(2):729-748. 
Table 1. Predicted PPRE sequences identified within $8.85 \mathrm{~kb}$ sequence upstream to Sost gene TSS using JASPAR position weighted matrix

\begin{tabular}{|c|c|c|c|c|c|}
\hline & Start & End & DNA Strand & Predicted sequence & Score \\
\hline PPRE1 & 32 & 46 & + & ATGAGGCAGAGGGGG & 8.5 \\
\hline PPRE2 & 666 & 680 & + & GTGGAGCTGGGGTCA & 8.9 \\
\hline PPRE3 & 1829 & 1843 & + & CAGGGGAAAAGGAGA & 9.2 \\
\hline PPRE4 & 1901 & 1915 & - & ACTTGGGAGAGGGCA & 8.7 \\
\hline PPRE5 & 3435 & 3449 & + & CTGGGTGAAAAGAGA & 8.5 \\
\hline PPRE6 & 3776 & 3790 & - & GGAGGCCAGAGGTCC & 12.5 \\
\hline PPRE7 & 3984 & 3998 & - & GCAGGGGCAGGGGCA & 9.2 \\
\hline PPRE8 & 3990 & 4004 & - & GCAGGGGCAGGGGCA & 9.2 \\
\hline PPRE9 & 4184 & 4198 & - & GTAGACTAGAGGCTA & 8.1 \\
\hline PPRE10 & 4277 & 4291 & + & GAGGCTCAGAGGGCA & 10.3 \\
\hline PPRE11 & 4593 & 4607 & - & GTGTGCTAAAGGCCA & 10.6 \\
\hline PPRE12 & 5331 & 5345 & - & TGGGGGCAGATTTCA & 8.4 \\
\hline PPRE13 & 7229 & 7243 & + & CTGAAGCAGAGTGGA & 9.1 \\
\hline PPRE14 & 7381 & 7395 & - & GTGGGGGAGGGGTCA & 15.3 \\
\hline PPRE15 & 7726 & 7740 & + & GGAGGGCAAGGTGAA & 9.6 \\
\hline
\end{tabular}

"Start" and "End" columns indicate PPRE bp position upstream of TSS. Bold text highlights PPREs chosen for further analysis. 